\title{
STRING-AVERAGING ALGORITHMS FOR CONVEX FEASIBILITY WITH INFINITELY MANY SETS
}

\author{
T. YUNG KONG, HOMEIRA PAJOOHESH, AND GABOR T. HERMAN
}

\begin{abstract}
Algorithms for convex feasibility find or approximate a point in the intersection of given closed convex sets. Typically there are only finitely many convex sets, but the case of infinitely many convex sets also has some applications. In this context, a string is a finite sequence of points each of which is obtained from the previous point by considering one of the convex sets. In a string-averaging algorithm, an iterative step from a first point to a second point computes a number of strings, all starting with the first point, and then calculates a (weighted) average of those strings' end-points: This average is that iterative step's second point, which is used as the first point for the next iterative step. For string-averaging algorithms based on strings in which each point either is the projection of the previous point on one of the convex sets or is equal to the previous point, we present theorems that provide answers to the following question: "How can the iterative steps be specified so that the string-averaging algorithm generates a convergent sequence whose limit lies in the intersection of the sets of a given convex feasibility problem?" This paper focuses on the case where the given collection of convex sets is infinite, whereas prior work on the same question that we are aware of has assumed the collection of convex sets is finite (or has been applicable only to a small subset of the algorithms we consider). The string-averaging algorithms that are shown to generate a convergent sequence whose limit lies in the intersection are also shown to be perturbation resilient, so they can be superiorized to generate sequences that also converge to a point of the intersection but in a manner that is superior with respect to some application-dependent criterion.
\end{abstract}

Keywords: convex feasibility, iterative algorithm, convergence, string-averaging, superiorization

\section{INTRODUCTION}

The convex feasibility problem is that of finding or approximating a point that belongs to every member of a collection of closed convex sets. This paper is concerned with the application of projection-based string-averaging (a methodology that will be further described in sec. 1.1) to the convex feasibility problem. Whereas most work on convex feasibility has assumed that the collection of convex sets has only finitely many members 1 our primary focus will instead be on the case where the collection is (countably) infinite.

Typical practical instances of the convex feasibility problem are inverse problems. The point we wish to find or approximate may, for example, be a point in $\mathbb{R}^{n}$ whose coordinates are the values of $n$ numerical attributes (sometimes referred to as "model parameters") of our model of some physical object, and the convex sets to which the point is assumed to belong may be determined by measurements taken of this object (sometimes referred to as "observed data"). In this context, the "forward" problem is to use our model to predict the values of the observed data from values of the $n$ model parameters. But our concern is the inverse

\footnotetext{
${ }^{1} \mathrm{~A}$ notable exception in the context of our results is the paper Comb97b], which studies a class of iterative schemes called $E M O P P$. It is shown in Comb97b that, under certain conditions, an EMOPP iterative scheme will converge to a solution of the convex feasibility problem for a finite or countably infinite collection of convex sets in a Hilbert space. However, most of the string-averaging iterative schemes considered in the present paper do not belong to the EMOPP class: Indeed, a string-averaging iterative scheme cannot belong to the EMOPP class unless the "strings" it uses all have length 1 (i.e., its weight functions satisfy condition $\mathrm{H} 2$ of sec. [1.2 with $m=1$ ) and its active averaging weights are bounded away from 0 . The results of Comb97b include results (for EMOPP iterative schemes) that are similar in nature to our first and third convergence theorems, but no results akin to our fourth or fifth convergence theorems.
} 
of that problem: Given the values of the observed data, find model parameters that are consistent with the data. This is a convex feasibility problem when the $n$ model parameters are regarded as the coordinates of a point $p$ in $\mathbb{R}^{n}$ and each observed data value constrains that point $p$ to lie in a certain closed convex subset of $\mathbb{R}^{n}$. For examples of such convex feasibility problems, see the papers CDH10, KSP09. Much other work that relates to convex feasibility can be found in the literature on inverse problems 2

As mentioned above, prior work on the convex feasibility problem has for the most part assumed the collection of convex sets is finite. Nevertheless, there has been some interest in convex feasibility problems with infinitely many convex sets. Indeed, the case of infinitely many sets is considered in some early seminal papers on the subject published more than two decades ago. For example, the 1967 paper of Gurin, Polyak and Raik GPR67] begins with: "MANY mathematical and applied problems can be reduced to finding some common point of a system (finite or infinite) of convex sets." Other examples are two 1997 papers by Combettes Comb97, Comb97b]: The paper Comb97b] was mentioned in footnote 1, while the paper Comb97. specifies that the collection of convex sets in its application of convex feasibility to image recovery may be "finite or countably infinite". The usefulness of the infinite case is demonstrated in YaOg04 by showing its applicability to set theoretic adaptive filtering schemes for nonstationary random processes. A rather more recent publication is Nedi10, in which the iterative algorithm for finding a point in the intersection of (infinitely many) given closed convex sets selects the $k+1$ st iterate by moving from the $k$ th iterate in the direction of the latter's projection on the $j$ th convex set, where $j$ is a stochastically selected index. In a broader context, there has been significant interest in algorithms that converge to common fixed points of infinite families of operators satisfying various conditions. Results relating to such algorithms are presented in, e.g., AlCe05, BaRZ18, BuRZ08, NiAb15, NiAb17, PhSu13, YYZ07].

The case of infinitely many sets is useful in applications where there is a potential infinity of samples or measurements, each one of which gives rise to a convex set that contains the point we wish to recover. For example, in [BlHe06] the problem of locating an acoustic source in a sensor network is formulated as a convex feasibility problem and necessary and sufficient conditions are given under which, when the number of samples increases to infinity, the convex feasibility problem has a unique solution at the true source location. In KSP09, it is shown that a particular algorithm of the kind we consider in the present paper is applicable to the problem of estimating the orientation distribution function (used to characterize crystallographic texture) from diffraction data. In one experiment discussed in KSP09, the algorithm is used to solve a convex feasibility problem in $\mathbb{R}^{1372000000}$ in which the number of convex sets is potentially infinite; each convex set is the set of points that satisfy a randomly generated equation, and an iterative step is carried out as soon as a new equation is available. The result reported in the paper for that experiment is the one obtained after 1000000000 iterations. While our theorems have no immediate application to the specific algorithm of [KSP09], the reported work demonstrates that the algorithms we consider in the present paper can be used to provide solutions even to enormously large problems.

\subsection{Notation and Definitions}

Let $C_{1}, C_{2}, C_{3}, \ldots$ be an infinite sequence of closed convex subsets of $\mathbb{R}^{n}$ (where $n$ is an arbitrary but fixed positive integer) such that $\bigcap_{i=1}^{\infty} C_{i}$ is nonempty and at least one of the sets $C_{i}$ is not $\mathbb{R}^{n}$. We write $\mathcal{C}$ to denote the collection of all the members of the sequence $\left(C_{i}\right)_{i=1}^{\infty}$. Thus $\mathcal{C}=\left\{C_{i} \mid 1 \leq i<\infty\right\}$ and $\mathbb{R}^{n} \supsetneq \bigcap \mathcal{C}=\bigcap_{i=1}^{\infty} C_{i} \neq \emptyset$. We use the term index to mean a positive integer that should be thought of as the index of one of the $C_{i}$ s.

We write $\|\boldsymbol{v}\|$ to denote the Euclidean norm of a vector $\boldsymbol{v}$, and for any $q \in \mathbb{R}^{n}$ and any nonempty set

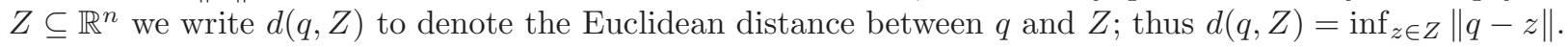

\footnotetext{
${ }^{2}$ At the time of writing, searching for the topic "convex feasibility" on the IOPScience website returns 30 articles, of which 24 were published in the journal Inverse Problems; a Google Scholar search for "convex feasibility" AND "inverse problems" returns many hundreds of articles.
} 
Since every member of $\mathcal{C}$ is closed and convex, for each $q \in \mathbb{R}^{n}$ and each index $j$ there will be a unique point $c \in C_{j}$ that minimizes $\|c-q\|$; we define $P_{j}: \mathbb{R}^{n} \rightarrow C_{j}$ to be the function that maps $q$ to that point of $C_{j}$. As a simple example, if $n=2$ and $C_{6}$ is the $y$-axis in $\mathbb{R}^{n}=\mathbb{R}^{2}$, then $P_{6}$ maps each point $(x, y) \in \mathbb{R}^{2}$ to the point $(0, y) \in C_{6}$. We call $P_{j}$ a projection operator. Note that $P_{j}(q)=q$ if and only if $q \in C_{j}$; an important special case of this is that if $C_{i}=\mathbb{R}^{n}$ then $P_{i}$ is the identity map.

Let $\mathbb{I}$ be the set of all nonempty finite sequences of indices. The members of $\mathbb{I}$ will be called index vectors. Note that $(i)$ is an index vector for every index $i$. If $\iota=\left(\iota_{1}, \iota_{2}, \ldots, \iota_{m}\right)$ is any index vector, then the integer $m$ will be denoted by Length $(\iota)$ and the set of indices $\left\{\iota_{1}, \iota_{2}, \ldots, \iota_{m}\right\}$ that occur in $\iota$ will be denoted by IndexSet $(\iota)$. The cardinality of the set $\operatorname{IndexSet}(\iota)$ cannot exceed Length $(\iota)$, and is exactly Length $(\iota)$ if and only if no two of the indices $\iota_{1}, \iota_{2}, \ldots, \iota_{m}$ are equal.

For any index vector $\iota=\left(\iota_{1}, \iota_{2}, \ldots, \iota_{m}\right)$ we write $P[\iota]$ to denote the operator $P_{\iota_{m}} \circ P_{\iota_{m-1}} \circ \cdots \circ P_{\iota_{1}}$; thus $P[\iota](x)=P_{\iota_{m}}\left(P_{\iota_{m-1}}\left(\ldots\left(P_{\iota_{1}}(x)\right) \ldots\right)\right) \in C_{\iota_{m}}$ for all $x \in \mathbb{R}^{n}$.

The results of this paper relate to the following question:

- How can we use operators of the form $P[\iota]$, or weighted averages of such operators, to iteratively generate a convergent sequence $x^{0}, x^{1}, x^{2}, \cdots \in \mathbb{R}^{n}$ whose limit lies in every member of $\mathcal{C}$ ?

Here $x^{0}$ is a user-specified "seed" point (which may be any point in $\mathbb{R}^{n}$ ), and the points $x^{1}, x^{2}, \ldots$ are to be computed one at a time, with $x^{k+1}$ being computed from the preceding point $x^{k}$ for $k=0,1,2, \ldots$ Each point $x^{k}$ will be called an iterate, and the process of computing $x^{k+1}$ from $x^{k}$ will be referred to as iteration $k$. In this context the integer $k \geq 0$ will be called the iteration number.

Our goal is to prove five convergence theorems. The first three of these theorems will concern sequences $x^{0}, x^{1}, x^{2}, \ldots$ in which each iterate $x^{k+1}$ is a weighted average of some finite subset of the set of points $\left\{P[\iota]\left(x^{k}\right) \mid \iota \in \mathbb{I}\right\}$. By this we mean that the iterates satisfy

$$
x^{k+1}=\sum_{\iota \in \mathbb{I}} w^{k}(\iota) P[\iota]\left(x^{k}\right) \quad \text { for all } k \geq 0
$$

for some functions $w^{0}, w^{1}, w^{2}, \cdots: \mathbb{I} \rightarrow[0,1]$ such that the following are true when $w$ is any $w^{k}$ :

A. $w$ has finite support-i.e., $\{\iota \in \mathbb{I} \mid w(\iota) \neq 0\}$ is a finite set.

B. $\sum_{\iota \in \mathbb{I}} w(\iota)=1$.

We will think of the sequence $x^{0}, x^{1}, x^{2}, \ldots$ defined by (1.1) as being generated by a string-averaging algorithm. Such algorithms were first proposed in CEH01, but only for the case of finitely many convex sets. In this context, a string is a finite sequence of points, each one of which is obtained from the previous point by considering exactly one of the convex sets. A string-averaging algorithm computes an iterate $x^{k+1}$ by first computing a finite number of strings, each starting with the preceding iterate $x^{k}$, and then calculates a weighted average of the last points of those strings; this weighted average is taken as $x^{k+1}$. Although CEH01 did not consider the possibility of using different weighted averaging operators at different iterations, other works have commonly allowed this BaRZ18, CeZa13, CeZa15, Comb97b, NiAb15, ReZa16, just as we will. Indeed, using the same weighted averaging operator at every iteration would be unsatisfactory when the collection $\mathcal{C}$ is infinite, as it would ignore all but finitely many of the members of $\mathcal{C}$.

Any function $w: \mathbb{I} \rightarrow[0,1]$ that satisfies conditions $\mathrm{A}$ and $\mathrm{B}$ will be called a weight function on $\mathbb{I}$ or just a weight function. If $w$ is a weight function and $\iota$ an index vector such that $w(\iota)>0$, we say $w$ uses $\iota$ and, for each index $i \in \operatorname{IndexSet}(\iota)$, we similarly say that $w$ uses $i$; we also refer to each member of the set $\{w(\iota) \mid \iota \in \mathbb{I}\} \backslash\{0\}$ as a weight or an averaging weight that is used by $w$. In the context of equation (1.1), we call $w^{k}$ the weight function used at iteration $k$, and say iteration $k$ uses an index vector $\iota$, an index $i$, or a weight $\mathrm{w}$ if $w^{k}$ uses $\iota, i$, or $\mathrm{w}$. If $k$ and $x^{k}$ are such that $w^{k}(\iota)=\mathrm{w}>0$ and $P[\iota] x^{k} \neq x^{k}$ for some index vector $\iota$, then we say the weight $\mathrm{w}$ is active at iteration $k$.

We do not assume the sets $C_{1}, C_{2}, C_{3}, \ldots$ are all known in advance; they may, for example, be presented to the algorithm one at a time. Similarly, we do not assume the weight functions $w^{0}, w^{1}, w^{2}, \ldots$ are fixed 
in advance. To compute $x^{k+1}$ from $x^{k}$ an algorithm might, for example, compute $P[\iota]\left(x^{k}\right)$ for various index vectors $\iota$ and then use the results to decide what weight function $w^{k}$ to use at iteration $k$. Moreover, the choice of $w^{k}$ need not be deterministic.

Before describing our theorems in more detail, we mention two noteworthy special cases of our results. The first special case, which we will call the finite case, is that in which there are only finitely many indices $i$ for which $C_{i} \neq \mathbb{R}^{n}$. In the finite case our convergence theorems can be understood as results about projection-based string-averaging algorithms for a finite sequence of sets $C_{1}, \ldots, C_{K}$.

The second special case is that in which $C_{1}=\mathbb{R}^{n}$ and $w^{k}((1))>0$ for at least one iteration number $k$. In this special case let us write $\lambda_{k}=1-w^{k}((1))$ for every iteration number $k$, so that $\lambda_{k}=\sum_{\iota \in \mathbb{I} \backslash\{(1)\}} w^{k}(\iota)$. Then our first three theorems may be construed as convergence theorems for under-relaxed versions of algorithms that generate sequences specified by (1.1); $\lambda_{k}$ is the relaxation parameter at iteration $k$. This is because we can rewrite (1.1) as

$$
x^{k+1}=x^{k}+\lambda_{k}\left(\left(\sum_{\iota \in \mathbb{I}} \omega^{k}(\iota) P[\iota]\left(x^{k}\right)\right)-x^{k}\right) \quad \text { for all } k \geq 0,
$$

where $\omega^{k}: \mathbb{I} \rightarrow[0,1]$ is a weight function such that $\lambda_{k} \omega^{k}(\iota)=w^{k}(\iota)$ for $\iota \in \mathbb{I} \backslash\{(1)\}$ and $\omega^{k}((1))=0$.

\subsection{Outline of the Main Results}

Our first three theorems imply that certain conditions (on $\mathcal{C}=\left\{C_{i} \mid 1 \leq i<\infty\right\}$ or on the weight functions $\left.w^{0}, w^{1}, w^{2}, \ldots\right)$ are sufficient for the sequence $x^{1}, x^{2}, x^{3} \ldots$ defined by equation (1.1) to converge to a point in $\bigcap \mathcal{C}$ (regardless of what point is chosen as the seed point $x^{0}$ ).

In the finite case, the second and third of these theorems are generalizations of the previously known fact that $x^{1}, x^{2}, x^{3} \ldots$ converges to a point in $\bigcap \mathcal{C}$ if the weight functions satisfy the following three additional hypotheses (which are not assumed in this paper):

H1. There is a positive lower bound on the nonzero averaging weights used by $\left(w^{k}\right)_{k=0}^{\infty}$-i.e., there is some $\epsilon>0$ such that $w^{k}(\iota)>\epsilon$ for every $k \geq 0$ and every $\iota \in \mathbb{I}$ such that $w^{k}(\iota) \neq 0$.

H2. There is an upper bound on the lengths of all the index vectors used by $\left(w^{k}\right)_{k=0}^{\infty}$-i.e., there is an integer $m$ such that, for every $k \geq 0$, if $\iota \in \mathbb{I}$ and $\operatorname{Length}(\iota)>m$ then $w^{k}(\iota)=0$.

H3. There is a positive integer $s$ such that each index $i$ for which $C_{i} \neq \mathbb{R}^{n}$ is used by at least one member of every $s$ consecutive members of the sequence $\left(w^{k}\right)_{k=0}^{\infty}$-i.e., there is a positive integer $s$ such that $\bigcup_{k=\kappa}^{\kappa+s-1}\left\{\operatorname{IndexSet}(\iota) \mid \iota \in \mathbb{I}\right.$ and $\left.w^{k}(\iota)>0\right\} \supseteq\left\{i \mid C_{i} \neq \mathbb{R}^{n}\right\}$ for every integer $\kappa \geq 0$.

That $x^{1}, x^{2}, x^{3} \ldots$ converges to a point in $\bigcap \mathcal{C}$ in the finite case if $\mathrm{H} 1-\mathrm{H} 3$ are all satisfied follows from [ReZa16. Thm. 4.1]; under the additional hypothesis that $\mathrm{H} 3$ holds with $s=1$, it also follows from CeZa13, Thm. 12]. Moreover, NiAb15, Prop. 3.8] implies that in the finite case $x^{1}, x^{2}, x^{3} \ldots$ converges to a point in $\bigcap \mathcal{C}$ whenever there are iteration numbers $n_{0}<n_{1}<n_{2}<\ldots$ such that, if we replaced $w^{k}$ with $w^{n_{k}}$ in $\mathrm{H} 1-\mathrm{H} 3$, then $\mathrm{H} 1$ and $\mathrm{H} 2$ would hold and $\mathrm{H} 3$ would hold with $s=1$.

But while the hypotheses H1 - H3 may seem quite mild in the finite case, when there are infinitely many indices $i$ for which $C_{i} \neq \mathbb{R}^{n}$ the hypothesis $\mathrm{H} 3$ cannot be satisfied - as each iteration uses only finitely many indices - and it would be quite restrictive to assume H1 or H2. (Hypothesis H1 would exclude the algorithm described in Example 1 of sec. 3.1. while H2 would exclude the algorithms described in Examples 2 and 3 of sec. [3.1.) Accordingly, whereas other authors have frequently assumed one or more of $\mathrm{H} 1$ - H3, none of these hypotheses will be assumed in this paper.

Our first convergence theorem assumes instead that $\bigcap \mathcal{C}$ is an $n$-dimensional subset of $\mathbb{R}^{n}$-i.e., $\cap \mathcal{C}$ contains an $n$-dimensional simplex. Under this hypothesis it says that, for any weight functions $w^{0}, w^{1}, w^{2}, \ldots$ and any seed point $x^{0}$, the sequence $x^{1}, x^{2}, x^{3} \ldots$ defined by equation (1.1) converges, and its limit lies in every $C_{i}$ for which the sum (over all iterations) of the weights of the index vectors that contain index $i$ is infinite. In particular, $x^{1}, x^{2}, x^{3} \ldots$ must converge to a point in $\bigcap \mathcal{C}$ if the weight functions satisfy the 
following condition: For every index $i$ such that $C_{i} \neq \mathbb{R}^{n}$, the sum (over all iterations) of the weights of the index vectors that contain index $i$ is infinite. (When $\mathrm{H} 1$ holds and $\mathrm{H} 2$ holds with $m=1$, this fact is a special case of Comb97b, Thm. 5.3(i)].)

The conclusions of the first convergence theorem do not always hold when the hypothesis that $\bigcap \mathcal{C}$ is an $n$ dimensional subset of $\mathbb{R}^{n}$ is not satisfied. However, our second convergence theorem gives sufficient conditions on $w^{0}, w^{1}, w^{2}, \ldots$ for $x^{1}, x^{2}, x^{3}, \ldots$ to converge to a point in $\bigcap \mathcal{C}$ even if the dimension of $\bigcap \mathcal{C}$ may be less than $n$. These sufficient conditions may be thought of as a greatly weakened version of the conditions H1 H3 above. We mention that the convergence of standard methods due to Cimmino CeEl02, Algorithm 2.1] (assuming the relaxation parameters $\lambda_{k}$ are all in $(0,1]$ and satisfy $\sum_{k=0}^{\infty} \lambda_{k}=\infty$ ) and to Kaczmarz [Kacz37. for solving finite systems of linear equations follows quite easily from the second convergence theorem.

The convergence of these standard methods will follow even more easily from our third convergence theorem, which assumes that the collection $\mathcal{C}$ satisfies the following additional condition:

$$
\operatorname{UASC}(\mathcal{C}): d(p, \bigcup\{C \in \mathcal{C} \mid p \notin C\})>0 \text { for all } p \in \mathbb{R}^{n} \backslash \cap \mathcal{C} \text {. }
$$

The condition $\mathbf{U A S C}(\mathcal{C})$ is so called because it holds just if the collection $\mathcal{C}$ has the property that the $\boldsymbol{u}$ nion of $\boldsymbol{a}$ ny $s$ ubcollection is closed. It is plain that $\mathbf{U A S C}(\mathcal{C})$ is always satisfied if $\mathcal{C}$ has only finitely many members, and this is probably the case of greatest practical interest.

However, UASC $(\mathcal{C})$ may hold even if $\mathcal{C}$ has infinitely many members. For example, UASC $(\mathcal{C})$ holds if the $C$ s form a descending chain in the sense that $C_{i} \supseteq C_{i+1}$ for every $i$. UASC $(\mathcal{C})$ also holds if each $C_{i}$ is the closed triangle in $\mathbb{R}^{2}$ whose vertices are $(-1,0),(1,0)$, and $\left(\cos \pi / 2^{i}, \sin \pi / 2^{i}\right)$. It is worth noting that if $\mathcal{C}$ and $\mathcal{C}^{\prime}$ are collections such that $\operatorname{UASC}(\mathcal{C})$ and $\mathbf{U A S C}\left(\mathcal{C}^{\prime}\right)$ hold, then $\operatorname{UASC}\left(\mathcal{C} \cup \mathcal{C}^{\prime}\right)$ holds too.

Our third convergence theorem says that if $\operatorname{UASC}(\mathcal{C})$ holds then the conclusion of the first convergence theorem holds regardless of the dimensionality of $\bigcap \mathcal{C}$ : For any weight functions $w^{0}, w^{1}, w^{2}, \ldots$ and any seed point $x^{0}$, the sequence $x^{1}, x^{2}, x^{3} \ldots$ defined by equation (1.1) converges, and its limit lies in every $C_{i}$ for which the sum (over all iterations) of the weights of the index vectors that contain index $i$ is infinite. In particular, $x^{1}, x^{2}, x^{3} \ldots$ must converge to a point in $\bigcap \mathcal{C}$ if $\mathbf{U A S C}(\mathcal{C})$ holds and there is no index $i$ such that $C_{i} \neq \mathbb{R}^{n}$ for which the sum (over all iterations) of the weights of the index vectors that contain index $i$ is finite. (When $\mathrm{H} 1$ holds and $\mathrm{H} 2$ holds with $m=1$, and $\mathcal{C}$ has only finitely many members, this fact is a special case of [Comb97b, Thm. 5.3(v)].)

Our fourth convergence theorem concerns "perturbed" versions of sequences $x^{0}, x^{1}, x^{2}, \ldots$ that satisfy equation (1.1). More precisely, the theorem relates to sequences $x_{*}^{0}, x_{*}^{1}, x_{*}^{2}, \cdots \in \mathbb{R}^{n}$ that satisfy

$$
x_{*}^{k+1}=\left(\sum_{\iota \in \mathbb{I}} w^{k}(\iota) P[\iota]\left(x_{*}^{k}\right)\right)+\boldsymbol{v}^{k} \quad \text { for all } k \geq 0
$$

for some weight functions $w^{0}, w^{1}, w^{2}, \cdots: \mathbb{I} \rightarrow[0,1]$, where each $\boldsymbol{v}^{k}$ is a vector in $\mathbb{R}^{n}$ that will be called the perturbation vector at iteration $k$.

The theorem makes two assertions which together say that, if the series $\sum_{i=0}^{\infty}\left\|\boldsymbol{v}^{k}\right\|$ converges, then the first three convergence theorems remain valid if we substitute an arbitrary sequence $x_{*}^{0}, x_{*}^{1}, x_{*}^{2}, \ldots$ that satisfies (1.2) for the sequence $x^{0}, x^{1}, x^{2}, \ldots$. If for every index $i$ such that $C_{i} \neq \mathbb{R}^{n}$ the sum (over all iterations) of the weights of the index vectors that contain index $i$ is infinite, and the hypotheses of one of the first three convergence theorems are satisfied, then the fourth convergence theorem tells us that an algorithm based on (1.1) has a property that is commonly referred to as (bounded) perturbation resilience - see, e.g., BaRZ18, CDH10, CeZa13, NiAb15, NiAb17, ReZa16 — with respect to the problem of finding a point in $\bigcap \mathcal{C}$. It follows that such algorithms are candidates for superiorization Cens15, CHJ17, a methodology for improving the efficacy of algorithms whose convergence is resilient to certain kinds of perturbation.

In the finite case, it is a previously known fact that our fourth convergence theorem holds when the hypotheses $\mathrm{H} 1$ - H3 above are all satisfied: That result follows from [ReZa16, Thm. 4.5]; under the additional hypothesis that $\mathrm{H} 3$ holds with $s=1$, it follows from [CeZa13, Thm. 12] as well. Under the hypotheses stated 
above in connection with NiAb15, Prop. 3.8], the result also follows from that proposition. But we are mainly interested in the case where $\mathcal{C}$ has infinitely many members, and so we do not assume H1 - H3.

Our fifth and last convergence theorem makes use of operators $P_{j}^{\epsilon}: \mathbb{R}^{n} \rightarrow \mathbb{R}^{n}$ that are defined for every $\epsilon>0$ and every index $j$ as follows:

$$
P_{j}^{\epsilon}(x)= \begin{cases}P_{j}(x) & \text { if }\left\|x-P_{j}(x)\right\| \geq \epsilon \\ x & \text { if }\left\|x-P_{j}(x)\right\|<\epsilon\end{cases}
$$

For any index vector $\iota=\left(\iota_{1}, \iota_{2}, \ldots, \iota_{m}\right)$, just as $P[\iota]$ denotes the composite operator $P_{\iota_{m}} \circ P_{\iota_{m}-1} \circ \cdots \circ P_{\iota_{1}}$, we write $P^{\epsilon}[\iota]$ to denote the operator $P_{\iota_{m}}^{\epsilon} \circ P_{\iota_{m-1}}^{\epsilon} \circ \cdots \circ P_{\iota_{1}}^{\epsilon}$. The fifth convergence theorem deals with sequences that are generated from a seed point in a way that is analogous to (1.2) but with $P^{\epsilon}[\iota]$ in place of $P[\iota]$ : It deals with sequences $x_{\epsilon}^{0}, x_{\epsilon}^{1}, x_{\epsilon}^{2}, \ldots$ that satisfy the condition

$$
x_{\epsilon}^{k+1}=\left(\sum_{\iota \in \mathbb{I}} w^{k}(\iota) P^{\epsilon}[\iota]\left(x_{\epsilon}^{k}\right)\right)+\boldsymbol{v}^{k} \quad \text { for all } k \geq 0
$$

for some weight functions $w^{0}, w^{1}, w^{2}, \cdots: \mathbb{I} \rightarrow[0,1]$ and perturbation vectors $\boldsymbol{v}^{0}, \boldsymbol{v}^{1}, \boldsymbol{v}^{2}, \cdots \in \mathbb{R}^{n}$.

The theorem says that if $\sum_{k=0}^{\infty}\left\|\boldsymbol{v}^{k}\right\|<\infty$ then, for any sequence of weight functions $w^{0}, w^{1}, w^{2}, \ldots$ and any seed point $x_{\epsilon}^{0}$, the sequence $x_{\epsilon}^{1}, x_{\epsilon}^{2}, x_{\epsilon}^{3}, \ldots$ defined by (1.3) converges, and its limit is within distance $\epsilon$ of every $C_{i}$ for which the sum (over all iterations) of the weights of the index vectors that contain index $i$ is infinite. This is true regardless of the dimensionality of $\bigcap \mathcal{C}$ and whether or not $\mathbf{U A S C}(\mathcal{C})$ holds.

Let us now assume that for every index $i$ such that $C_{i} \neq \mathbb{R}^{n}$ the sum (over all iterations) of the weights of the index vectors that contain index $i$ is infinite. Then it follows from this fifth convergence theorem that, for any positive integers $m$ and $N$ and any seed point $y^{0} \in \mathbb{R}^{n}$, we can compute successive points in the sequence defined by (1.3) with some $\epsilon<1 / N$ and $x_{\epsilon}^{0}=y^{0}$ until we find a point $y^{1}$ such that $\max _{1 \leq i \leq m} d\left(y^{1}, C_{i}\right) \leq 1 / N$. We can repeat this process with $y^{1}$ as seed point (instead of $y^{0}$ ), new perturbation vectors, and larger values of $m$ and $N$ to find a point $y^{2}$, which we use as the next seed point. Repeating this process with larger and larger values of $m$ and $N$, we obtain a sequence $y^{3}, y^{4}, y^{5}, \ldots$ that must in fact converge to a point that lies in every member of $\mathcal{C}$, assuming the sum of the magnitudes of the perturbation vectors used in computing this sequence is finite 3

\section{First Convergence Theorem}

In the rest of this paper, $w^{0}, w^{1}, w^{2}, \ldots$ will denote arbitrary weight functions on $\mathbb{I}$, and $x^{0}, x^{1}, x^{2}, \ldots$ points in $\mathbb{R}^{n}$ that satisfy equation (1.1) for all iteration numbers $k \geq 0$. For each index $i$ the set of all the index vectors in which $i$ occurs will be denoted by $\mathbb{I}\langle i\rangle$. Thus $\mathbb{I}\langle i\rangle=\{\iota \in \mathbb{I} \mid i \in \operatorname{IndexSet}(\iota)\}$. For example, $(3,1,4,3,1,3,7) \in \mathbb{I}\langle i\rangle$ just if $i=1,3,4$, or 7 . Note that $\mathbb{I}\langle i\rangle$ is an infinite set for every index $i$.

Recall from the Introduction that $\mathcal{C}=\left\{C_{i} \mid 1 \leq i<\infty\right\}$, where each $C_{i}$ is a closed convex subset of $\mathbb{R}^{n}$, $\bigcap_{i=1}^{\infty} C_{i}=\bigcap \mathcal{C} \neq \emptyset$, and there is at least one index $i$ for which $C_{i} \neq \mathbb{R}^{n}$. The main theorem of this section is:

Theorem 2.1 (First Convergence Theorem). Suppose $\bigcap \mathcal{C}$ is an $n$-dimensional subset of $\mathbb{R}^{n}$ (i.e., $\bigcap \mathcal{C}$ contains an $n$-dimensional simplex). Then the sequence $x^{0}, x^{1}, x^{2}, \ldots$ converges, and its limit lies in $C_{j}$ for every index $j$ such that $\sum_{k=0}^{\infty} \sum_{\iota \in \mathbb{I}\langle j\rangle} w^{k}(\iota)=\infty$. In particular, the sequence $x^{0}, x^{1}, x^{2}, \ldots$ converges to a point in $\bigcap \mathcal{C}$ if $\sum_{k=0}^{\infty} \sum_{\iota \in \mathbb{I}\langle j\rangle} w^{k}(\iota)=\infty$ for every index $j$ such that $C_{j} \neq \mathbb{R}^{n}$.

This theorem will be proved in sec. 2.3 .

\footnotetext{
${ }^{3}$ If $l_{m}$ is the number of iterations of (1.3) that are carried out to compute $y^{m+1}$ from $y^{m}$, and $\left(\boldsymbol{v}_{m}^{k}\right)_{k=0}^{l_{m}-1}$ is the sequence of perturbation vectors that are used by those $l_{m}$ iterations, then what we are assuming here is that $\sum_{m=0}^{\infty} \sum_{k=0}^{l_{m}-1}\left\|\boldsymbol{v}_{m}^{k}\right\|<\infty$.
} 


\subsection{Counterexamples When $\bigcap \mathcal{C}$ is Not $n$-Dimensional}

The conclusions of the First Convergence Theorem may be false if $\bigcap \mathcal{C}$ is not an $n$-dimensional subset of $\mathbb{R}^{n}$ : Even if $\sum_{k=0}^{\infty} \sum_{\iota \in \mathbb{I}\langle i\rangle} w^{k}(\iota)=\infty$ for every index $i$, a sequence $\left(x^{k}\right)_{k=0}^{\infty}$ that satisfies (1.1) may fail to converge or may converge to a point that is not in $\bigcap \mathcal{C}=\bigcap_{i=1}^{\infty} C_{i}$.

Example 4.1 of Comb97b provides a counterexample in which $\left(x^{k}\right)_{k=0}^{\infty}$ fails to converge even though $\sum_{k=0}^{\infty} \sum_{\iota \in \mathbb{I}\langle i\rangle} w^{k}(\iota)=\infty$ for every index $i$. That counterexample is an instance of a class of counterexamples we now describe.

Let $\left(\theta_{i}\right)_{i=0}^{\infty}$ be any infinite sequence of real numbers that are not all equal for which the following conditions all hold:

1. $0 \leq\left|\theta_{i+1}-\theta_{i}\right|<\pi / 2$ for $0 \leq i<\infty$.

2. As $i \rightarrow \infty,\left|\theta_{i+1}-\theta_{i}\right|$ is $O(1 / i)$ (which implies $\log \cos \left(\theta_{i+1}-\theta_{i}\right)$ is $O\left(1 / i^{2}\right)$, by Taylor's Theorem).

3. For each index $i$ there are infinitely many indices $j$ such that $\theta_{j} \bmod 2 \pi=\theta_{i} \bmod 2 \pi$.

Bearing in mind that $\sum_{i=1}^{\infty} 1 / i=\infty$, it is easy to construct such sequences $\left(\theta_{i}\right)_{i=0}^{\infty}$. For example, writing $\phi_{i}$ for $\sum_{j=1}^{i} 1 / j$, we can obtain such a sequence $\left(\theta_{i}\right)_{i=0}^{\infty}$ from the sequence $\left(\phi_{i}\right)_{i=0}^{\infty}$ by inserting additional numbers in such a way that the resulting sequence satisfies condition 3 as well as conditions 1 and 24

Now let $R_{1}, R_{2}, R_{3}, \cdots \subset \mathbb{R}^{2}$ be the sequence of closed rays starting from $(0,0)$ such that each ray $R_{i}$ passes through the point $\left(\cos \theta_{i}, \sin \theta_{i}\right)$. (Thus $R_{i}=R_{j}$ if and only if $\theta_{i} \bmod 2 \pi=\theta_{j} \bmod 2 \pi$.) Let $x^{0}$ be the point $\left(\cos \theta_{0}, \sin \theta_{0}\right)$, and for $0 \leq k<\infty$ let $x^{k+1}$ be the orthogonal projection of $x^{k}$ on $R_{k+1}$.

Let $\left(C_{i}\right)_{i=1}^{\infty}$ be the sequence obtained from $\left(R_{i}\right)_{i=1}^{\infty}$ by omitting all but the first occurrence of each ray in $\left(R_{i}\right)_{i=1}^{\infty}$, so that $C_{i} \neq C_{j}$ whenever $i \neq j$ and $\left\{C_{i} \mid 1 \leq i<\infty\right\}=\left\{R_{i} \mid 1 \leq i<\infty\right\}$. For $1 \leq i<\infty$, let $\alpha(i)$ be the index such that $R_{i}=C_{\alpha(i)}$. This implies $x^{k+1}=P_{\alpha(k+1)}\left(x^{k}\right)$ for $0 \leq k<\infty$ (where $P_{j}: \mathbb{R}^{n} \rightarrow C_{j}$ is the projection map, as before). So if for every iteration number $k$ we define

$$
w^{k}(\iota)= \begin{cases}1 & \text { if } \iota=(\alpha(k+1)) \\ 0 & \text { otherwise }\end{cases}
$$

then $\left(x^{k}\right)_{k=0}^{\infty}$ satisfies equation (1.1). Moreover, for every index $i$ it follows from (2.1) that $\sum_{\iota \in \mathbb{I}\langle i\rangle} w^{k}(\iota)=1$ whenever $\alpha(k+1)=i$ (i.e., whenever $R_{k+1}=C_{i}$ ), and hence that $\sum_{k=0}^{\infty} \sum_{\iota \in \mathbb{I}\langle i\rangle} w^{k}(\iota)=\infty$ (as condition 3 above implies there are infinitely many $k \mathrm{~s}$ for which $\left.R_{k+1}=C_{i}\right)$. However, $\left(x^{k}\right)_{k=0}^{\infty}$ fails to converge: Indeed, as $k \rightarrow \infty$ it follows from conditions 1 and 2 that $\left\|x^{k}\right\|=\prod_{i=0}^{k-1} \cos \left(\theta_{i+1}-\theta_{i}\right)$ and that this product converges to a nonzero value (since $\sum_{i=1}^{\infty} 1 / i^{2}$ converges), while conditions 1 and 3 imply $\theta_{k}$ mod $2 \pi$ does not converge.

We can give a similar counterexample to show that a sequence satisfying (1.1) may converge to a point that is not in $\bigcap_{i=1}^{\infty} C_{i}$ even though $\sum_{k=0}^{\infty} \sum_{\iota \in \mathbb{I}\langle i\rangle} w^{k}(\iota)=\infty$ for every index $i$. Let $y^{0}, y^{1}, y^{2}, \ldots$ be a convergent subsequence $x^{r_{0}}, x^{r_{1}}, x^{r_{2}}, \ldots$ of the sequence $x^{0}, x^{1}, x^{2}, \ldots$ of the above counterexample, and redefine the weight function $w^{k}$ for each $k$ so that $w^{k}(\iota)=1$ if $\iota=\left(\alpha\left(r_{k}+1\right), \alpha\left(r_{k}+2\right), \ldots, \alpha\left(r_{k+1}\right)\right)$ and $w^{k}(\iota)=0$ otherwise. Now the convergent sequence $y^{0}, y^{1}, y^{2}, \ldots$ satisfies equation (1.1) with $y^{k+1}$ and $y^{k}$ in place of $x^{k+1}$ and $x^{k}$. Much as before, we have that $\sum_{k=0}^{\infty} \sum_{\iota \in \mathbb{I}\langle i\rangle} w^{k}(\iota)=\infty$ for every index $i$. But if $y^{\infty}=$ $\lim _{k \rightarrow \infty} y^{k}$ then we have that $\left\|y^{\infty}\right\|=\lim _{k \rightarrow \infty}\left\|y^{k}\right\|=\lim _{k \rightarrow \infty}\left\|x^{k}\right\|>0$, and so $y^{\infty} \notin \bigcap_{i=1}^{\infty} C_{i}=\{(0,0)\}$.

In the above counterexamples the collection $\mathcal{C}=\left\{C_{i} \mid 1 \leq i<\infty\right\}$ has infinitely many members. We will see from the Third Convergence Theorem that there are no such counterexamples in the finite case.

\footnotetext{
${ }^{4}$ One way to do this is to let $\left(\theta_{i}\right)_{i=0}^{\infty}$ be the nondecreasing sequence obtained from $\left(\phi_{i}\right)_{i=0}^{\infty}$ by inserting the sequence of numbers $\left(\phi_{j} \bmod 2 \pi+(2 j+1) 2^{k} \pi\right)_{k=1}^{\infty}$ for each $j \geq 0$. Then $\left(\theta_{i}\right)_{i=0}^{\infty}$ satisfies conditions 1 and 3 . For each positive even integer $2 m$ there exist unique integers $j \geq 0$ and $k \geq 1$ such that $(2 j+1) 2^{k}=2 m$ (as is clear when we write $2 m$ in binary notation), and so the interval $[2 m \pi, 2(m+1) \pi)$ contains exactly one of the inserted numbers. It can be seen from this that the sequence $\left(\theta_{i}\right)_{i=0}^{\infty}$ satisfies condition 2 .
} 


\subsection{Preliminary Results}

We now establish some basic properties of the projection operators $P_{i}$ and the sequence $x^{0}, x^{1}, x^{2}, \ldots$ that will be used to prove the First Convergence Theorem. In this subsection we do not assume $\bigcap \mathcal{C}$ is an $n$-dimensional subset of $\mathbb{R}^{n}$, so the results we establish here can also be used to prove other convergence theorems that do not have this hypothesis.

The results of this subsection depend on the following geometrical fact:

Lemma 2.2. Let $u, v$, and $a$ be points in $\mathbb{R}^{n}$ such that $(a-u) \cdot(v-u)>0$. Then the line segment that joins $u$ to $v$ contains points that are closer to a than $u$ is.

Proof. When $0 \leq \epsilon \leq 1$ the point $u+\epsilon(v-u)$ lies on the closed line segment that joins $u$ to $v$, and for sufficiently small positive $\epsilon$ it is readily confirmed that this point is closer to $a$ than $u$ is: Indeed, the squares of the distances from $a$ to $u$ and from $a$ to $u+\epsilon(v-u)$ are respectively $\|a-u\|^{2}$ and $\|a-u-\epsilon(v-u)\|^{2}$, and we have that $\|a-u\|^{2}-\|a-u-\epsilon(v-u)\|^{2}=2 \epsilon(a-u) \cdot(v-u)-\epsilon^{2}\|v-u\|^{2}>0$ when $\epsilon$ is sufficiently small and positive $($ since $(a-u) \cdot(v-u)>0)$.

Proposition 2.3. Let $i$ be an index such that $C_{i} \neq \mathbb{R}^{n}$, let $p \in C_{i}$, and let $z \in \mathbb{R}^{n}$. Then:

$$
\begin{aligned}
\left\|P_{i}(z)-p\right\|^{2} & \leq\|z-p\|^{2}-\left\|P_{i}(z)-z\right\|^{2}-2 d\left(p, \mathbb{R}^{n} \backslash C_{i}\right)\left\|P_{i}(z)-z\right\| \\
& =\|z-p\|^{2}-d\left(z, C_{i}\right)^{2}-2 d\left(p, \mathbb{R}^{n} \backslash C_{i}\right) d\left(z, C_{i}\right)
\end{aligned}
$$

Proof. We have that $\left\|P_{i}(z)-p\right\|^{2}=\|z-p\|^{2}-\left\|P_{i}(z)-z\right\|^{2}-2\left(P_{i}(z)-p\right) \cdot\left(z-P_{i}(z)\right)$ because:

$$
\|z-p\|^{2}=\left\|\left(P_{i}(z)-p\right)+\left(z-P_{i}(z)\right)\right\|^{2}=\left\|P_{i}(z)-p\right\|^{2}+\left\|z-P_{i}(z)\right\|^{2}+2\left(P_{i}(z)-p\right) \cdot\left(z-P_{i}(z)\right)
$$

So it remains only to show that:

$$
\left(P_{i}(z)-p\right) \cdot\left(z-P_{i}(z)\right) \geq d\left(p, \mathbb{R}^{n} \backslash C_{i}\right)\left\|z-P_{i}(z)\right\|
$$

Note that $\left(P_{i}(z)-p\right) \cdot\left(z-P_{i}(z)\right) \geq 0$, for otherwise it would follow from Lemma 2.2 (on putting $a=z$, $u=P_{i}(z)$, and $v=p$ ) that the line segment joining $p$ to $P_{i}(z)$ contains points that are strictly closer to $z$ than $P_{i}(z)$ is, which would contradict the definition of $P_{i}(z)$ since the line segment lies in the convex set $C_{i}$.

If $z \in C_{i}$, then $P_{i}(z)=z$ and (2.2) is true. Now let us assume $z \notin C_{i}$. Let $y$ be the orthogonal projection of $p$ on the hyperplane through $P_{i}(z)$ that is orthogonal to $z-P_{i}(z)$. Then $\left(z-P_{i}(z)\right) \cdot\left(y-P_{i}(z)\right)=0$, and so $\left(z-P_{i}(z)\right) \cdot\left(y+\lambda\left(z-P_{i}(z)\right)-P_{i}(z)\right)>0$ for all $\lambda>0$. By Lemma 2.2, this implies that for all $\lambda>0$ the line segment with endpoints $P_{i}(z)$ and $y+\lambda\left(z-P_{i}(z)\right)$ contains points that are strictly closer to $z$ than $P_{i}(z)$ is. Such points are not in $C_{i}$ (as $P_{i}(z)$ is the point in $C_{i}$ that is closest to $z$ ), so we see from the convexity of $C_{i}$ (and the fact that $\left.P_{i}(z) \in C_{i}\right)$ that $y+\lambda\left(z-P_{i}(z)\right) \notin C_{i}$ if $\lambda>0$. Thus, there exist points arbitrarily close to $y$ that are not in $C_{i}$, whence $d\left(p, \mathbb{R}^{n} \backslash C_{i}\right) \leq\|y-p\|$. Moreover, it follows from the definition of $y$ that the vector projection of $P_{i}(z)-p$ on $z-P_{i}(z)$ is $y-p$, and we have seen that $\left(P_{i}(z)-p\right) \cdot\left(z-P_{i}(z)\right) \geq 0$. Hence $\left(P_{i}(z)-p\right) \cdot \frac{z-P_{i}(z)}{\left\|z-P_{i}(z)\right\|}=\|y-p\| \geq d\left(p, \mathbb{R}^{n} \backslash C_{i}\right)$, which implies (2.2).

We now deduce a few lemmas from Proposition 2.3 .

Lemma 2.4. Let $\iota=\left(\iota_{1}, \ldots, \iota_{m}\right) \in \mathbb{I}$ be such that $\bigcap_{j \in \operatorname{IndexSet}(\iota)} C_{j}=\bigcap_{j=1}^{m} C_{\iota_{j}} \neq \mathbb{R}^{n}$, let $s \in \bigcap_{j=1}^{m} C_{\iota_{j}}$, and let $z \in \mathbb{R}^{n}$. Then:

1. $\|P[\iota](z)-s\| \leq\|z-s\|$, with equality just if $z \in \bigcap_{j=1}^{m} C_{\iota_{j}}$.

2. $\|P[\iota](z)-s\|^{2} \leq\|z-s\|^{2}-2 d\left(s, \mathbb{R}^{n} \backslash \bigcap_{j=1}^{m} C_{\iota_{j}}\right)\|P[\iota](z)-z\|$.

3. $\|P[\iota](z)-s\|^{2} \leq\|z-s\|^{2}-2 d\left(s, \mathbb{R}^{n} \backslash \bigcap_{j=1}^{m} C_{\iota_{j}}\right) d\left(z, C_{\iota_{q}}\right)$ for $1 \leq q \leq m$. 
Proof. We see from Proposition 2.3 that if $p \in C_{i}$ and $z \in \mathbb{R}^{n}$ then $\left\|P_{i}(z)-p\right\| \leq\|z-p\|$, with equality just if $z \in C_{i}$. Assertion 1 follows from applying this observation to each $P_{\iota_{j}}$ in turn, since $s \in C_{\iota_{j}}$ for $1 \leq j \leq m$.

To prove assertions 2 and 3 , we define $z_{0}=z$ and, for $1 \leq r \leq m$, we define $z_{r}=P\left[\left(\iota_{1}, \iota_{2}, \ldots, \iota_{r}\right)\right](z)$, so that $z_{m}=P[\iota](z)$. Since

$$
d\left(s, \mathbb{R}^{n} \backslash \bigcap_{j=1}^{m} C_{\iota_{j}}\right) \leq d\left(s, \mathbb{R}^{n} \backslash C_{\iota_{r}}\right),
$$

the following is true for $1 \leq r \leq m$ :

$$
\begin{array}{rlr}
\left\|z_{r}-s\right\|^{2} & =\left\|P_{\iota_{r}}\left(z_{r-1}\right)-s\right\|^{2} & \\
& \leq\left\|z_{r-1}-s\right\|^{2}-2 d\left(s, \mathbb{R}^{n} \backslash C_{\iota_{r}}\right)\left\|z_{r}-z_{r-1}\right\| & \text { by Proposition 2.3 } \\
& \leq\left\|z_{r-1}-s\right\|^{2}-2 d\left(s, \mathbb{R}^{n} \backslash \bigcap_{j=1}^{m} C_{\iota_{j}}\right)\left\|z_{r}-z_{r-1}\right\| &
\end{array}
$$

Hence the following holds for $1 \leq q \leq m$ :

$$
\begin{aligned}
\left\|z_{q}-s\right\|^{2} & \leq\left\|z_{0}-s\right\|^{2}-2 d\left(s, \mathbb{R}^{n} \backslash \bigcap_{j=1}^{m} C_{\iota_{j}}\right) \sum_{r=1}^{q}\left\|z_{r}-z_{r-1}\right\| \\
& \leq\left\|z_{0}-s\right\|^{2}-2 d\left(s, \mathbb{R}^{n} \backslash \bigcap_{j=1}^{m} C_{\iota_{j}}\right)\left\|z_{q}-z_{0}\right\|,
\end{aligned}
$$

where the second inequality follows from the triangle inequality. Since $z_{0}=z$ and $z_{m}=P[\iota](z)$, we get assertion 2 from the case $q=m$ of (2.4).

Since $z_{q} \in C_{\iota_{q}}$, we have that $\left\|z_{q}-z_{0}\right\|=\left\|z_{q}-z\right\| \geq d\left(z, C_{\iota_{q}}\right)$. This and (2.4) imply

$$
\left\|z_{q}-s\right\|^{2} \leq\left\|z_{0}-s\right\|^{2}-2 d\left(s, \mathbb{R}^{n} \backslash \bigcap_{j=1}^{m} C_{\iota_{j}}\right) d\left(z, C_{\iota_{q}}\right) \quad \text { for } 1 \leq q \leq m .
$$

Assertion 3 follows, as $\|P[\iota](z)-s\|=\left\|z_{m}-s\right\| \leq\left\|z_{q}-s\right\|$ for $1 \leq q \leq m$ (by (2.3) $)$.

We see from equation (1.1), and conditions $\mathrm{A}$ and $\mathrm{B}$ of the definition of a weight function on $\mathbb{I}$, that if $g: \mathbb{R}^{n} \rightarrow \mathbb{R}$ is any convex function then

$$
g\left(x^{k+1}\right) \leq \sum_{\iota \in \mathbb{I}} w^{k}(\iota) g\left(P[\iota]\left(x^{k}\right)\right) \quad \text { for all } k \geq 0
$$

by Jensen's Inequality for functions on $\mathbb{R}^{n}$ Webs94, Sec. 5.4].

It is an easy consequence of the triangle inequality that $z \mapsto\|z-a\|$ is a convex function on $\mathbb{R}^{n}$ for any $a \in \mathbb{R}^{n}$. It follows that $z \mapsto\|z-a\|^{2}$ is also a convex function on $\mathbb{R}^{n}$ (because $z \mapsto z^{2}$ is a convex function on $\mathbb{R}$ ). On applying (2.5) to the function $g(z)=\|z-a\|^{2}$, we deduce that:

$$
\left\|x^{k+1}-a\right\|^{2} \leq \sum_{\iota \in \mathbb{I}} w^{k}(\iota)\left\|P[\iota]\left(x^{k}\right)-a\right\|^{2} \quad \text { for all } k \geq 0 \text { and all } a \in \mathbb{R}^{n} .
$$

We use this to prove:

Lemma 2.5. Let $c \in \bigcap \mathcal{C}$. Then for every iteration number $k$ we have that:

$$
\left\|x^{k+1}-c\right\|^{2} \leq\left\|x^{k}-c\right\|^{2}-2 d\left(c, \mathbb{R}^{n} \backslash \bigcap \mathcal{C}\right)\left\|x^{k+1}-x^{k}\right\|
$$

Proof. If $\iota=\left(\iota_{1}, \ldots, \iota_{m}\right)$, then $d\left(c, \mathbb{R}^{n} \backslash \bigcap \mathcal{C}\right) \leq d\left(c, \mathbb{R}^{n} \backslash \bigcap_{j=1}^{m} C_{\iota_{j}}\right)$. Bearing this in mind, and writing $\delta$ for $d\left(c, \mathbb{R}^{n} \backslash \bigcap \mathcal{C}\right)$, we have that:

$$
\begin{aligned}
\left\|x^{k+1}-c\right\|^{2} & \leq \sum_{\iota \in \mathbb{I}} w^{k}(\iota)\left\|P[\iota]\left(x^{k}\right)-c\right\|^{2} & & \text { by [2.6) } \\
& \leq \sum_{\iota \in \mathbb{I}} w^{k}(\iota)\left(\left\|x^{k}-c\right\|^{2}-2 \delta\left\|P[\iota]\left(x^{k}\right)-x^{k}\right\|\right) & & \text { by statement 2 of Lemma 2.4 } \\
& =\left\|x^{k}-c\right\|^{2}-2 \delta \sum_{\iota \in \mathbb{I}} w^{k}(\iota)\left\|P[\iota]\left(x^{k}\right)-x^{k}\right\| & & \text { as } \sum_{\iota \in \mathbb{I}} w^{k}(\iota)=1 \\
& \leq\left\|x^{k}-c\right\|^{2}-2 \delta\left\|\left(\sum_{\iota \in \mathbb{I}} w^{k}(\iota) P[\iota]\left(x^{k}\right)\right)-x^{k}\right\| & & \text { by (2.5) } \\
& =\left\|x^{k}-c\right\|^{2}-2 \delta\left\|x^{k+1}-x^{k}\right\| & &
\end{aligned}
$$

for all iteration numbers $k$. 
Corollary 2.6. Let $c \in \bigcap \mathcal{C}$. Then we have that

$$
\left\|x^{r}-c\right\|^{2} \leq\left\|x^{0}-c\right\|^{2}-2 d\left(c, \mathbb{R}^{n} \backslash \bigcap \mathcal{C}\right) \sum_{k=0}^{r-1}\left\|x^{k+1}-x^{k}\right\|
$$

for all iteration numbers $r$.

Lemma 2.7. Let $c \in \bigcap \mathcal{C}$. Then for every iteration number $k$ we have that:

$$
\left\|x^{k+1}-c\right\|^{2} \leq\left\|x^{k}-c\right\|^{2}-2 d\left(c, \mathbb{R}^{n} \backslash \cap \mathcal{C}\right) d\left(x^{k}, C_{j}\right) \sum_{\iota \in \mathbb{I}\langle j\rangle} w^{k}(\iota) \quad \text { for all indices } j .
$$

Proof. Let us write $\delta$ for $d\left(c, \mathbb{R}^{n} \backslash \cap \mathcal{C}\right)$. Then for every index $j$ we have that

$$
\begin{array}{rlrl}
\left\|x^{k+1}-c\right\|^{2} \leq & \sum_{\iota \in \mathbb{I}} w^{k}(\iota)\left\|P[\iota]\left(x^{k}\right)-c\right\|^{2} & \text { by [2.6]) } \\
& =\sum_{\iota \in \mathbb{I}\langle j\rangle} w^{k}(\iota)\left\|P[\iota]\left(x^{k}\right)-c\right\|^{2} & \\
& \quad+\sum_{\iota \notin \mathbb{I}\langle j\rangle} w^{k}(\iota)\left\|P[\iota]\left(x^{k}\right)-c\right\|^{2} & \\
\leq & \sum_{\iota \in \mathbb{I}\langle j\rangle} w^{k}(\iota)\left(\left\|x^{k}-c\right\|^{2}-2 d\left(x^{k}, C_{j}\right) \delta\right) & \\
& \quad+\sum_{\iota \notin \mathbb{I}\langle j\rangle} w^{k}(\iota)\left\|x^{k}-c\right\|^{2} & & \text { by statements } 1 \& 3 \text { of Lemma 2.4 } \\
& =\left\|x^{k}-c\right\|^{2}-2 d\left(x^{k}, C_{j}\right) \delta \sum_{\iota \in \mathbb{I}\langle j\rangle} w^{k}(\iota) & & \text { as } \sum_{\iota \in \mathbb{I}} w^{k}(\iota)=1
\end{array}
$$

for all iteration numbers $k$.

Corollary 2.8. For all indices $j$, iteration numbers $r$, and $c \in \bigcap \mathcal{C}$ we have that

$$
\left\|x^{r}-c\right\|^{2} \leq\left\|x^{0}-c\right\|^{2}-2 d\left(c, \mathbb{R}^{n} \backslash \cap \mathcal{C}\right) \sum_{k=0}^{r-1} \sum_{\iota \in \mathbb{I}\langle j\rangle} d\left(x^{k}, C_{j}\right) w^{k}(\iota) .
$$

Hence $\sum_{k=0}^{\infty} \sum_{\iota \in \mathbb{I}\langle j\rangle} d\left(x^{k}, C_{j}\right) w^{k}(\iota)<\infty$ for every index $j$ if $d\left(c, \mathbb{R}^{n} \backslash \cap \mathcal{C}\right)>0$ for some $c \in \bigcap \mathcal{C}$.

\subsection{Proof of the First Convergence Theorem}

Under the hypotheses of the theorem $\bigcap \mathcal{C}$ contains an $n$-dimensional simplex. Let $c$ be the barycenter of some $n$-simplex in $\bigcap \mathcal{C}$, so that $d\left(c, \mathbb{R}^{n} \backslash \bigcap \mathcal{C}\right)>0$. Then it follows from Corollary 2.6 (and the fact that $\left\|x^{r}-c\right\|^{2} \geq 0$ for all $r$ ) that the series $\sum_{k=0}^{\infty}\left\|x^{k+1}-x^{k}\right\|$ must converge.

Since $\left\|x^{r_{2}}-x^{r_{1}}\right\| \leq \sum_{k=r_{1}}^{r_{2}-1}\left\|x^{k+1}-x^{k}\right\|$ for all iteration numbers $r_{1}<r_{2}$ (by the triangle inequality), and since $\sum_{k=0}^{\infty}\left\|x^{k+1}-x^{k}\right\|$ converges, $x^{0}, x^{1}, x^{2}, \ldots$ is a Cauchy sequence and must therefore converge.

Let $x^{\infty}$ be the limit of the sequence and $j$ any index for which $x^{\infty} \notin C_{j}$, so that

$$
d\left(x^{k}, C_{j}\right)>d\left(x^{\infty}, C_{j}\right) / 2>0 \text { for all sufficiently large } k .
$$

Then the series $\sum_{k=0}^{\infty} \sum_{\iota \in \mathbb{I}\langle j\rangle} w^{k}(\iota)$ converges, for if this series were divergent then it would follow from (2.7) that the series $\sum_{k=0}^{\infty} \sum_{\iota \in \mathbb{I}\langle j\rangle} d\left(x^{k}, C_{j}\right) w^{k}(\iota)$ also diverges, contrary to Corollary 2.8

\section{Second Convergence Theorem}

As we saw in subsection 2.1 the First Convergence Theorem would not be true if we dropped the hypothesis that $\bigcap \mathcal{C}$ is an $n$-dimensional subset of $\mathbb{R}^{n}$. However, the convergence theorem we establish in the present section will not depend on this hypothesis.

As before, $w^{0}, w^{1}, w^{2}, \ldots$ will denote arbitrary weight functions on $\mathbb{I}$, and $x^{0}, x^{1}, x^{2}, \ldots$ points in $\mathbb{R}^{n}$ that satisfy equation (1.1) for all iteration numbers $k \geq 0$. For any weight function $w$, we define

$$
\operatorname{MaxLength}(w)=\max \{\operatorname{Length}(\iota) \mid \iota \in \mathbb{I} \text { and } w(\iota)>0\}
$$

If $\iota=\left(\iota_{1}, \iota_{2}, \ldots, \iota_{m}\right) \in \mathbb{I}$ and $i \in \operatorname{IndexSet}(\iota)=\left\{\iota_{1}, \iota_{2}, \ldots, \iota_{m}\right\}$, then we define $\operatorname{Pos}(i, \iota)$ to be the least $j$ for which $\iota_{j}=i$. If $i \notin \operatorname{IndexSet}(\iota)$, then we say $\operatorname{Pos}(i, \iota)=\infty$. As examples, if $\iota=(9,8,2,4,1,6,2,1,2)$ 
then $\operatorname{Pos}(1, \iota)=5, \operatorname{Pos}(2, \iota)=3, \operatorname{Pos}(3, \iota)=\infty$, and $\operatorname{Pos}(4, \iota)=4$. Note that $\operatorname{Pos}(i, \iota)>0$, and that $\operatorname{Pos}(i, \iota) \leq \operatorname{Length}(\iota)$ whenever $\iota \in \mathbb{I}\langle i\rangle$.

For any real numbers $f_{1}, f_{2}, f_{3}, \cdots \in[0,1]$ such that $\sum_{r=1}^{\infty} f_{r}=\infty$, the following convergence theorem gives a condition on the weight functions $w^{0}, w^{1}, w^{2}, \ldots$ that is sufficient to ensure that $x^{0}, x^{1}, x^{2}, \ldots$ converges to a point in $\bigcap \mathcal{C}$. Provided $f_{1}, f_{2}, f_{3}, \cdots \in[0,1]$ and $\sum_{r=1}^{\infty} f_{r}=\infty$, the smaller the numbers $f_{1}, f_{2}, f_{3}, \ldots$ the better (i.e., the more general) the corresponding sufficient condition will be. For example, the sufficient condition given by $f_{r}=\frac{1}{100 r \log (r+1)}$ is rather better than the sufficient condition given by $f_{r}=\frac{1}{10 r}$.

Theorem 3.1 (Second Convergence Theorem). Let $f_{1}, f_{2}, f_{3}, \cdots \in[0,1]$ be numbers such that $\sum_{r=1}^{\infty} f_{r}=\infty$. Suppose there is an infinite strictly increasing sequence of iteration numbers $\kappa_{1}<\kappa_{2}<\kappa_{3}<\ldots$ such that both of the following are true:

C1: $\sum_{k=\kappa_{r}}^{\kappa_{r+1}-2} \operatorname{MaxLength}\left(w^{k}\right) \leq \frac{1}{f_{r}}$ for all $r \in \mathbb{Z}^{+}$such that $\kappa_{r+1} \geq \kappa_{r}+2$ and $f_{r}>0$.

C2: For every index $i$ such that $C_{i} \neq \mathbb{R}^{n}, \max _{\kappa_{r} \leq k<\kappa_{r+1}} \sum_{\iota \in \mathbb{I}\langle i\rangle} \frac{w^{k}(\iota)}{\operatorname{Pos}(i, \iota)} \geq f_{r}$ for all sufficiently large $r \in \mathbb{Z}^{+}$. Then $x^{0}, x^{1}, x^{2}, \ldots$ converges to a point in $\bigcap \mathcal{C}$.

As C1 is vacuously satisfied if $\kappa_{r+1}=\kappa_{r}+1$ for all $r \in \mathbb{Z}^{+}$, the special case of this theorem in which $\kappa_{r}=r$ for all $r \in \mathbb{Z}^{+}$can be stated more simply, as follows:

Corollary 3.2. Let $f_{1}, f_{2}, f_{3}, \cdots \in[0,1]$ be such that $\sum_{r=1}^{\infty} f_{r}=\infty$, and suppose the following is true for every index $i$ such that $C_{i} \neq \mathbb{R}^{n}$ :

$$
\sum_{\iota \in \mathbb{I}\langle i\rangle} \frac{w^{r}(\iota)}{\operatorname{Pos}(i, \iota)} \geq f_{r} \quad \text { for all sufficiently large } r \in \mathbb{Z}^{+} .
$$

Then $x^{0}, x^{1}, x^{2}, \ldots$ converges to a point in $\bigcap \mathcal{C}$.

\subsection{Examples}

Before proving the Second Convergence Theorem, we give simple examples of weight functions for which the theorem implies convergence of the sequence of iterates $x^{0}, x^{1}, x^{2}, \ldots$ to a point in $\bigcap \mathcal{C}$.

In the finite case, it is easy to see that if the weight functions satisfy the hypotheses H1, H2, and H3 of sec. 1.2 then $\mathbf{C} 1$ and $\mathbf{C 2}$ hold for $\left(\kappa_{r}\right)_{r=1}^{\infty}=s, 2 s, 3 s, 4 s, \ldots$ and $\left(f_{r}\right)_{r=0}^{\infty}=d, d, d, d, \ldots$, where $s$ is any sufficiently large positive integer and $d$ is any sufficiently small positive constant. But in the finite case we will see from the Third Convergence Theorem that the condition " $\sum_{k=0}^{\infty} \sum_{\iota \in \mathbb{I}\langle j\rangle} w^{k}(\iota)=\infty$ for every index $j$ such that $C_{j} \neq \mathbb{R}^{n}$ " is sufficient for $x^{0}, x^{1}, x^{2}, \ldots$ to converge to a point in $\bigcap \mathcal{C}$, and this is a much more general sufficient condition than the ones given by the Second Convergence Theorem.

In the rest of this subsection we give three examples which do not assume $\mathcal{C}$ has only finitely many members. The first two examples are analogs for infinite collections of commonly used procedures for converging to the intersection of a finite collection of closed convex sets. In Example $1, x^{k+1}$ is defined in a way that is reminiscent of Cimmino's method Cimm38 of computing approximate solutions of systems of linear equations (and especially of Algorithm 2.1 of [CeEl02 with unity relaxation). Similarly, the iterates of Example 2 are defined in a way that is reminiscent of Kaczmarz's method [Kacz37] of computing approximate solutions of linear equation systems.

Example 1. Suppose the sequence of iterates $x^{0}, x^{1}, x^{2}, x^{3}, \ldots$ satisfies

$$
x^{k+1}=\frac{1}{k} P_{1}\left(x^{k}\right)+\frac{1}{k} P_{2}\left(x^{k}\right)+\cdots+\frac{1}{k} P_{k}\left(x^{k}\right) \quad \text { for all } k \geq 1 .
$$


When $r \geq 1$, we have that $w^{r}(\iota)>0$ just if $\iota$ is one of the $r$ index vectors $(1),(2), \ldots,(r)$. For any index $i$, when $r \geq i$ there is just one $\iota \in \mathbb{I}\langle i\rangle$ for which $w^{r}(\iota)>0$, namely $\iota=(i)$, and we have that $w^{r}(\iota)=1 / r$ and $\operatorname{Pos}(i, \iota)=1$ for this $\iota$. Hence $\sum_{\iota \in \mathbb{I}\langle i\rangle} \frac{w^{r}(\iota)}{\operatorname{Pos}(i, \iota)}=1 / r$ if $r \geq i$, and so the hypotheses of Corollary 3.2 are satisfied for $f_{r}=1 / r$. Therefore $\left(x^{k}\right)_{k=0}^{\infty}$ converges to a point in $\bigcap \mathcal{C}$.

Example 2. For every iteration number $k \geq 1$, let $\iota^{k}$ be some permutation of $(1,2, \ldots, k)$. Suppose the sequence of iterates $x^{0}, x^{1}, x^{2}, x^{3}, \ldots$ satisfies

$$
x^{k+1}=P\left[\iota^{k}\right]\left(x^{k}\right) \quad \text { for all } k \geq 1 .
$$

When $r \geq 1$, we have that $w^{r}(\iota)>0$ just if $\iota=\iota^{r}$. For this $\iota$, we have that $w^{r}(\iota)=1$ and when $r \geq i$ we have that $\iota \in \mathbb{I}\langle i\rangle$, and $\operatorname{Pos}(i, \iota) \leq r$. Hence $\sum_{\iota \in \mathbb{I}\langle i\rangle} \frac{w^{r}(\iota)}{\operatorname{Pos}(i, \iota)} \geq 1 / r$ if $r \geq i$. So the hypotheses of Corollary 3.2 are satisfied for $f_{r}=1 / r$, and $\left(x^{k}\right)_{k=0}^{\infty}$ converges to a point in $\bigcap \mathcal{C}$.

Example 3. For odd iteration numbers $k \geq 1$, let $\iota^{k}$ be some permutation of $(1,3,5, \ldots, k)$. For even iteration numbers $k \geq 2$, let $\iota^{k}$ be some permutation of $(2,4,6, \ldots, k)$. Suppose

$$
x^{k+1}=\frac{1}{2} P_{k+2}\left(x^{k}\right)+\frac{1}{2} P\left[\iota^{k}\right]\left(x^{k}\right) \text { for all } k \geq 1 .
$$

Let us verify that the hypotheses of the Second Convergence Theorem are satisfied when we put $\kappa_{r}=2 r$ and $f_{r}=\frac{1}{2(r+1)}$ for all $r \geq 1$.

Now $\mathbf{C} 1$ holds because:

$$
\sum_{k=\kappa_{r}}^{\kappa_{r+1}-2} \operatorname{MaxLength}\left(w^{k}\right)=\sum_{k=2 r}^{2 r} \operatorname{MaxLength}\left(w^{k}\right)=\operatorname{MaxLength}\left(w^{2 r}\right)=\operatorname{Length}\left(\iota^{2 r}\right)=r<\frac{1}{f_{r}}
$$

It remains to verify $\mathbf{C 2}$.

As $\kappa_{r}=2 r$ and $f_{r}=\frac{1}{2(r+1)}, \mathbf{C} 2$ is equivalent to the statement that for every index $j$ there exists an $r_{j} \in \mathbb{Z}^{+}$such that at least one of the following is true for all $r \geq r_{j}$ :

$$
\sum_{\iota \in \mathbb{I}\langle j\rangle} \frac{w^{2 r}(\iota)}{\operatorname{Pos}(j, \iota)} \geq \frac{1}{2(r+1)} \quad \text { or } \quad \sum_{\iota \in \mathbb{I}\langle j\rangle} \frac{w^{2 r+1}(\iota)}{\operatorname{Pos}(j, \iota)} \geq \frac{1}{2(r+1)}
$$

When $j$ is even this holds because if $\iota=\iota^{2 r}$ and $2 r \geq j$, then $\iota \in \mathbb{I}\langle j\rangle, w^{2 r}(\iota)=\frac{1}{2}$, and $\operatorname{Pos}(j, \iota) \leq r$, whence $\frac{w^{2 r}(\iota)}{\operatorname{Pos}(j, \iota)}>\frac{1}{2(r+1)}$. When $j$ is odd it holds because if $\iota=\iota^{2 r+1}$ and $2 r+1 \geq j$, then $\iota \in \mathbb{I}\langle j\rangle, w^{2 r+1}(\iota)=\frac{1}{2}$, and $\operatorname{Pos}(j, \iota) \leq r+1$, whence $\frac{w^{2 r+1}(\iota)}{\operatorname{Pos}(j, \iota)} \geq \frac{1}{2(r+1)}$. Therefore $\mathbf{C 2}$ holds.

So $\left(x^{k}\right)_{k=0}^{\infty}$ converges to a point in $\bigcap \mathcal{C}$.

\subsection{Proof of the Second Convergence Theorem}

As a consequence of Lemma 2.5 (which implies $\left\|x^{k+1}-c\right\| \leq\left\|x^{k}-c\right\|$ for any $c \in \bigcap \mathcal{C}$ and all iteration numbers $k$ ), we deduce:

Lemma 3.3. If $x^{0}, x^{1}, x^{2}, \ldots$ has a subsequence that converges to a point $c$ in $\bigcap \mathcal{C}$, then $x^{0}, x^{1}, x^{2}, \ldots$ converges to $c$.

Our proof of the Second Convergence Theorem will be based on the following claim:

Claim 3.4. To show that the sequence of iterates $x^{0}, x^{1}, x^{2}, \ldots$ converges to a point in $\bigcap \mathcal{C}$, it is enough to show that for every index $q$ there is an integer $k_{q}$ for which the following is true:

$$
\max _{1 \leq i \leq q} d\left(x^{k_{q}}, C_{i}\right) \leq 1 / q
$$


Justification of Claim 3.4. Suppose that for every index $q$ there is an integer $k_{q}$ for which (3.1) is true. As $x^{k_{1}}, x^{k_{2}}, x^{k_{3}}, \ldots$ is a bounded sequence in $\mathbb{R}^{n}$ (by Lemma 2.5), it has a convergent subsequence. For every index $q$ we see from (3.1) that $d\left(x^{k_{r}}, C_{q}\right) \leq 1 / r$ for all integers $r \geq q$, which implies that if $x$ is the limit of a convergent subsequence of $x^{k_{1}}, x^{k_{2}}, x^{k_{3}}, \ldots$ then $d\left(x, C_{q}\right) \leq 1 / r$ for all positive integers $r$ (i.e., $\left.d\left(x, C_{q}\right)=0\right)$, whence $x \in C_{q}$ (since $C_{q}$ is closed). Thus the limit of a convergent subsequence of $x^{k_{1}}, x^{k_{2}}, x^{k_{3}}, \ldots$ is a point in $\bigcap \mathcal{C}$, and so it follows from Lemma 3.3 that $x^{0}, x^{1}, x^{2}, \ldots$ converges to that same point.

To show there is a sequence of iteration numbers $k_{1}<k_{2}<k_{3}<\ldots$ such that (3.1) holds for every index $q$, we use the inequalities stated in the following lemma and its corollary:

Titu's Lemma. Let $m$ be any integer greater than or equal to $2, z_{1}, \ldots, z_{m}$ any sequence of $m$ real numbers, and $\lambda_{1}, \ldots, \lambda_{m}$ any sequence of $m$ positive real numbers. Then:

$$
\frac{z_{1}^{2}}{\lambda_{1}}+\cdots+\frac{z_{m}^{2}}{\lambda_{m}} \geq \frac{\left(z_{1}+\cdots+z_{m}\right)^{2}}{\lambda_{1}+\cdots+\lambda_{m}}
$$

Proof. This well known inequality is equivalent to the Cauchy-Schwarz inequality for the pair of vectors $\left(z_{1} / \sqrt{\lambda_{1}}, \ldots, z_{m} / \sqrt{\lambda_{m}}\right)$ and $\left(\sqrt{\lambda_{1}}, \ldots, \sqrt{\lambda_{m}}\right)$.

Corollary. Let $m$ be any integer greater than or equal to 2, and $z_{1}, \ldots, z_{m}$ any sequence of $m$ real numbers. Then we have that $z_{1}^{2}+\cdots+z_{m}^{2} \geq\left(z_{1}+\cdots+z_{m}\right)^{2} / m$.

We now proceed to show the existence of iteration numbers $k_{1}<k_{2}<k_{3}<\ldots$ that satisfy (3.1) for every index $q$.

Lemma 3.5. Let $c$ be any point in $\bigcap \mathcal{C}$. Let $y \in \mathbb{R}^{n}$, and let $\iota=\left(\iota_{1}, \iota_{2}, \ldots, \iota_{m}\right)$ be any index vector. Let $y_{0}=y$, and, for $1 \leq j \leq m$, let $y_{j}=P\left[\left(\iota_{1}, \iota_{2}, \ldots, \iota_{j}\right)\right](y)$ and let $d_{j}=\left\|y_{j}-y_{j-1}\right\|=d\left(y_{j-1}, C_{\iota_{j}}\right)$. Then for $1 \leq j \leq m$ we have that:

$$
\begin{gathered}
\left\|y_{j}-c\right\|^{2} \leq\left\|y_{j-1}-c\right\|^{2}-d_{j}^{2} \\
\left\|y_{j}-c\right\|^{2} \leq\|y-c\|^{2}-\left(d_{1}^{2}+\cdots+d_{j}^{2}\right) \\
\left\|y_{j}-y\right\| \leq d_{1}+\cdots+d_{j} \\
\left\|y_{j}-c\right\|^{2} \leq\|y-c\|^{2}-\left\|y_{j}-y\right\|^{2} / j \\
\|P[\iota](y)-c\|^{2}=\left\|y_{m}-c\right\|^{2} \leq\left\|y_{j}-c\right\|^{2} \leq\|y-c\|^{2}-d\left(y, C_{\iota_{j}}\right)^{2} / j
\end{gathered}
$$

Proof. Since $y_{j}=P_{\iota_{j}}\left(y_{j-1}\right)$, (3.2) follows from Proposition 2.3 (3.3) follows from (3.2). Inequality (3.4) follows from the triangle inequality. Inequality (3.5) follows from (3.3), the corollary to Titu's Lemma, and (3.4). The first inequality of (3.6) follows from the fact that (3.2) holds for $1 \leq j \leq m$. The second inequality of (3.6) follows from (3.5) and the fact that $\left\|y_{j}-y\right\| \geq d\left(y, C_{\iota_{j}}\right)$ because $y_{j} \in C_{\iota_{j}}$.

Lemma 3.6. Let $c \in \cap \mathcal{C}$, let $i$ be any index, and let $k$ be any iteration number. Then:

$$
\left\|x^{k+1}-c\right\|^{2} \leq\left\|x^{k}-c\right\|^{2}-d\left(x^{k}, C_{i}\right)^{2} \sum_{\iota \in \mathbb{I}\langle i\rangle} \frac{w^{k}(\iota)}{\operatorname{Pos}(i, \iota)}
$$

Proof. On putting $j=\operatorname{Pos}(i, \iota)$ in (3.6), we see that if $\iota \in \mathbb{I}\langle i\rangle$ and $y \in \mathbb{R}^{n}$ then:

$$
\|P[\iota](y)-c\|^{2} \leq\|y-c\|^{2}-\frac{d\left(y, C_{i}\right)^{2}}{\operatorname{Pos}(i, \iota)}
$$


Moreover, for any $y \in \mathbb{R}^{n}$ we see from (3.6) that $\|P[\iota](y)-c\|^{2} \leq\|y-c\|^{2}$ even if $\iota \notin \mathbb{I}\langle i\rangle$. From this, (3.7), and (2.6) we deduce that:

$$
\begin{aligned}
\left\|x^{k+1}-c\right\|^{2} & \leq \sum_{\iota \in \mathbb{I}\langle i\rangle} w^{k}(\iota)\left\|P[\iota]\left(x^{k}\right)-c\right\|^{2}+\sum_{\iota \notin \mathbb{I}\langle i\rangle} w^{k}(\iota)\left\|P[\iota]\left(x^{k}\right)-c\right\|^{2} \\
& \leq \sum_{\iota \in \mathbb{I}\langle i\rangle} w^{k}(\iota)\left(\left\|x^{k}-c\right\|^{2}-\frac{d\left(x^{k}, C_{i}\right)^{2}}{\operatorname{Pos}(i, \iota)}\right)+\sum_{\iota \notin \mathbb{I}\langle i\rangle} w^{k}(\iota)\left\|x^{k}-c\right\|^{2}
\end{aligned}
$$

Here the right side is equal to $\left\|x^{k}-c\right\|^{2}-d\left(x^{k}, C_{i}\right)^{2} \sum_{\iota \in \mathbb{I}\langle i\rangle} \frac{w^{k}(\iota)}{\operatorname{Pos}(i, \iota)}$ because $\sum_{\iota \in \mathbb{I}} w^{k}(\iota)=1$.

Lemma 3.7. Let $c \in \bigcap \mathcal{C}$ and let $k$ be any iteration number. Then we have that:

$$
\left\|x^{k+1}-c\right\|^{2} \leq\left\|x^{k}-c\right\|^{2}-\frac{\left\|x^{k+1}-x^{k}\right\|^{2}}{\operatorname{MaxLength}\left(w^{k}\right)}
$$

Proof. For each index vector $\iota$ used at iteration $k$, Length $(\iota) \leq \operatorname{MaxLength}\left(w^{k}\right)$ and so (3.5) implies $\left\|P[\iota]\left(x^{k}\right)-c\right\|^{2} \leq\left\|x^{k}-c\right\|^{2}-\left\|P[\iota]\left(x^{k}\right)-x^{k}\right\|^{2} / \operatorname{MaxLength}\left(w^{k}\right)$ or, equivalently,

$$
\left\|P[\iota]\left(x^{k}\right)-c\right\|^{2}+\left\|P[\iota]\left(x^{k}\right)-x^{k}\right\|^{2} / \operatorname{MaxLength}\left(w^{k}\right) \leq\left\|x^{k}-c\right\|^{2}
$$

As $u \mapsto\|u-c\|^{2}$ and $u \mapsto\left\|u-x^{k}\right\|^{2}$ are convex functions on $\mathbb{R}^{n}$, we have that:

$$
\begin{array}{rlr}
\left\|x^{k+1}-c\right\|^{2} & +\frac{\left\|x^{k+1}-x^{k}\right\|^{2}}{\text { MaxLength }\left(w^{k}\right)} \\
& =\left\|\left(\sum_{\iota \in \mathbb{I}} w^{k}(\iota) P[\iota]\left(x^{k}\right)\right)-c\right\|^{2}+\frac{\left\|\left(\sum_{\iota \in \mathbb{I}} w^{k}(\iota) P[\iota]\left(x^{k}\right)\right)-x^{k}\right\|^{2}}{\operatorname{MaxLength}\left(w^{k}\right)} & \\
& \leq \sum_{\iota \in \mathbb{I}} w^{k}(\iota)\left\|P[\iota]\left(x^{k}\right)-c\right\|^{2}+\frac{\sum_{\iota \in \mathbb{I}} w^{k}(\iota)\left\|P[\iota]\left(x^{k}\right)-x^{k}\right\|^{2}}{\operatorname{MaxLength}\left(w^{k}\right)} & \text { by (2.5) } \\
& =\sum_{\iota \in \mathbb{I}} w^{k}(\iota)\left(\left\|P[\iota]\left(x^{k}\right)-c\right\|^{2}+\left\|P[\iota]\left(x^{k}\right)-x^{k}\right\|^{2} / \operatorname{MaxLength}\left(w^{k}\right)\right) & \\
& \leq \sum_{\iota \in \mathbb{I}} w^{k}(\iota)\left\|x^{k}-c\right\|^{2} & \text { by (3.9) } \\
& =\left\|x^{k}-c\right\|^{2} & \text { as } \sum_{\iota \in \mathbb{I}} w^{k}(\iota)=1
\end{array}
$$

which immediately implies (3.8).

Corollary 3.8. Let $\kappa<\kappa^{*}$ be iteration numbers and let $c \in \bigcap \mathcal{C}$. Then:

$$
\left\|x^{\kappa^{*}}-c\right\|^{2} \leq\left\|x^{\kappa}-c\right\|^{2}-\frac{\left\|x^{\kappa^{*}}-x^{\kappa}\right\|^{2}}{\sum_{k=\kappa}^{\kappa^{*}-1} \operatorname{MaxLength}\left(w^{k}\right)}
$$

Proof. We have that

$$
\begin{aligned}
& \left\|x^{\kappa^{*}}-c\right\|^{2} \leq\left\|x^{\kappa}-c\right\|^{2}-\sum_{k=\kappa}^{\kappa^{*}-1} \frac{\left\|x^{k+1}-x^{k}\right\|^{2}}{\operatorname{MaxLength}\left(w^{k}\right)} \quad \text { by Lemma } 3.7 \\
& \leq\left\|x^{\kappa}-c\right\|^{2}-\frac{\left(\sum_{k=\kappa}^{\kappa^{*}-1}\left\|x^{k+1}-x^{k}\right\|\right)^{2}}{\sum_{k=\kappa}^{\kappa^{*}-1} \text { MaxLength }\left(w^{k}\right)} \quad \text { by Titu's Lemma } \\
& \leq\left\|x^{\kappa}-c\right\|^{2}-\frac{\left\|x^{\kappa^{*}}-x^{\kappa}\right\|^{2}}{\sum_{k=\kappa}^{\kappa^{*}-1} \operatorname{MaxLength}\left(w^{k}\right)} \quad \text { by the triangle inequality }
\end{aligned}
$$

which establishes (3.10). 
Lemma 3.9. Let $c \in \cap \mathcal{C}$, let $\kappa \leq \kappa^{*}<\kappa^{\prime}$ be iteration numbers, and let $i$ be any index. Then

$$
\left\|x^{\kappa^{\prime}}-c\right\|^{2} \leq\left\|x^{\kappa}-c\right\|^{2}-\min \left(\frac{1}{\sum_{k=\kappa}^{\kappa^{*}-1} \operatorname{MaxLength}\left(w^{k}\right)}, \sum_{\iota \in \mathbb{I}\langle i\rangle} \frac{w^{\kappa^{*}}(\iota)}{\operatorname{Pos}(i, \iota)}\right) \frac{d\left(x^{\kappa}, C_{i}\right)^{2}}{4},
$$

where $\min \left(\frac{1}{\sum_{k=\kappa}^{\kappa^{*}-1} \operatorname{MaxLength}\left(w^{k}\right)}, \sum_{\iota \in \mathbb{I}\langle i\rangle} \frac{w^{\kappa^{*}}(\iota)}{\operatorname{Pos}(i, \iota)}\right)$ should be understood to mean $\sum_{\iota \in \mathbb{I}\langle i\rangle} \frac{w^{\kappa^{*}}(\iota)}{\operatorname{Pos}(i, \iota)}$ if $\kappa=\kappa^{*}$.

Proof. We first consider the case in which $\left\|x^{\kappa^{*}}-x^{\kappa}\right\|>d\left(x^{\kappa}, C_{i}\right) / 2$. In this case $\kappa<\kappa^{*}<\kappa^{\prime}$ and so

$$
\begin{array}{rlrl}
\left\|x^{\kappa^{\prime}}-c\right\|^{2} & \leq\left\|x^{\kappa^{*}}-c\right\|^{2} & & \text { by Lemma 2.5 } \\
& \leq\left\|x^{\kappa}-c\right\|^{2}-\frac{\left\|x^{\kappa^{*}}-x^{\kappa}\right\|^{2}}{\sum_{k=\kappa}^{\kappa^{*}-1} \operatorname{MaxLength}\left(w^{k}\right)} & \text { by Corollary 3.8 } \\
& <\left\|x^{\kappa}-c\right\|^{2}-\left(\frac{1}{\sum_{k=\kappa}^{\kappa^{*}-1} \operatorname{MaxLength}\left(w^{k}\right)}\right) \frac{d\left(x^{\kappa}, C_{i}\right)^{2}}{4} & \text { as }\left\|x^{\kappa^{*}}-x^{\kappa}\right\|>d\left(x^{\kappa}, C_{i}\right) / 2
\end{array}
$$

and the lemma holds.

Now we consider the case in which $\left\|x^{\kappa^{*}}-x^{\kappa}\right\| \leq d\left(x^{\kappa}, C_{i}\right) / 2$. In this case we see from the triangle inequality that $d\left(x^{\kappa^{*}}, C_{i}\right) \geq d\left(x^{\kappa}, C_{i}\right) / 2$, whence

$$
d\left(x^{\kappa^{*}}, C_{i}\right)^{2} \geq \frac{d\left(x^{\kappa}, C_{i}\right)^{2}}{4}
$$

Therefore, since $\kappa \leq \kappa^{*}<\kappa^{\prime}$, we have that

$$
\begin{array}{rlr}
\left\|x^{\kappa^{\prime}}-c\right\|^{2} & \leq\left\|x^{\kappa^{*}+1}-c\right\|^{2} & \text { by Lemma 2.5 } \\
& \leq\left\|x^{\kappa^{*}}-c\right\|^{2}-d\left(x^{\kappa^{*}}, C_{i}\right)^{2} \sum_{\iota \in \mathbb{I}\langle i\rangle} \frac{w^{\kappa^{*}}(\iota)}{\operatorname{Pos}(i, \iota)} & \text { by Lemma 3.6 } \\
& \leq\left\|x^{\kappa^{*}}-c\right\|^{2}-\frac{d\left(x^{\kappa}, C_{i}\right)^{2}}{4} \sum_{\iota \in \mathbb{I}\langle i\rangle} \frac{w^{\kappa^{*}}(\iota)}{\operatorname{Pos}(i, \iota)} & \text { by [3.11) } \\
& \leq\left\|x^{\kappa}-c\right\|^{2}-\frac{d\left(x^{\kappa}, C_{i}\right)^{2}}{4} \sum_{\iota \in \mathbb{I}\langle i\rangle} \frac{w^{\kappa^{*}}(\iota)}{\operatorname{Pos}(i, \iota)} & \text { by Lemma 2.5 }
\end{array}
$$

and so the lemma holds in this case too.

Completion of the Proof of the Second Convergence Theorem. Suppose the hypotheses of the theorem are satisfied, so that $f_{1}, f_{2}, f_{3}, \cdots \in[0,1]$ satisfy $\sum_{r=1}^{\infty} f_{r}=\infty$ and $\kappa_{1}<\kappa_{2}<\kappa_{3}<\ldots$ are iteration numbers such that

C1: $\sum_{k=\kappa_{r}}^{\kappa_{r+1}-2}$ MaxLength $\left(w^{k}\right) \leq 1 / f_{r}$ for all $r \in \mathbb{Z}^{+}$such that $\kappa_{r+1} \geq \kappa_{r}+2$ and $f_{r}>0$.

C2: For every index $i$ such that $C_{i} \neq \mathbb{R}^{n}, \max _{\kappa_{r} \leq k<\kappa_{r+1}} \sum_{\iota \in \mathbb{I}\langle i\rangle} \frac{w^{k}(\iota)}{\operatorname{Pos}(i, \iota)} \geq f_{r}$ for all sufficiently large $r \in \mathbb{Z}^{+}$. For every index $i$ such that $C_{i} \neq \mathbb{R}^{n}$ and all $r \in \mathbb{Z}^{+}$, let $\kappa_{r}^{*}(i)$ be an iteration number such that $\kappa_{r} \leq \kappa_{r}^{*}(i)<$ $\kappa_{r+1}$ and $\kappa_{r}^{*}(i) \in \underset{\kappa_{r} \leq k<\kappa_{r+1}}{\arg \max } \sum_{\iota \in \mathbb{I}\langle i\rangle} \frac{w^{k}(\iota)}{\operatorname{Pos}(i, \iota)}$. [Here $\underset{\kappa_{r} \leq k<\kappa_{r+1}}{\arg \max } \sum_{\iota \in \mathbb{I}\langle i\rangle} \frac{w^{k}(\iota)}{\operatorname{Pos}(i, \iota)}$ denotes the set of those iteration numbers $k$ that maximize $\sum_{\iota \in \mathbb{I}\langle i\rangle} \frac{w^{k}(\iota)}{\operatorname{Pos}(i, \iota)}$ subject to the condition $\kappa_{r} \leq k<\kappa_{r+1}$.] 
For every index $i$ such that $C_{i} \neq \mathbb{R}^{n}, \kappa_{r}^{*}(i)<\kappa_{r+1}$ implies

$$
\sum_{k=\kappa_{r}}^{\kappa_{r}^{*}(i)-1} \operatorname{MaxLength}\left(w^{k}\right) \leq \sum_{k=\kappa_{r}}^{\kappa_{r+1}-2} \operatorname{MaxLength}\left(w^{k}\right)
$$

and so we see from $\mathbf{C} 1$ and $\mathbf{C 2}$ that

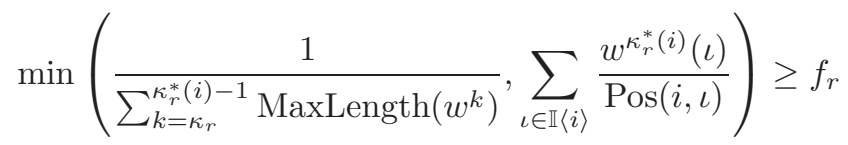

if $r$ is sufficiently large, where the left side should be understood to mean $\sum_{\iota \in \mathbb{I}\langle i\rangle} \frac{w^{\kappa_{r}^{*}(i)}(\iota)}{\operatorname{Pos}(i, \iota)}$ if $\kappa_{r}=\kappa_{r}^{*}(i)$.

For every index $i$ such that $C_{i} \neq \mathbb{R}^{n}$, it follows from Lemma 3.9 (on putting $\left(\kappa, \kappa^{*}, \kappa^{\prime}\right)=\left(\kappa_{r}, \kappa_{r}^{*}(i), \kappa_{r+1}\right)$ ), and the fact that (3.12) holds for all sufficiently large $r$, that

$$
\left\|x^{\kappa_{r+1}}-c\right\|^{2} \leq\left\|x^{\kappa_{r}}-c\right\|^{2}-\frac{f_{r}}{4} d\left(x^{\kappa_{r}}, C_{i}\right)^{2}
$$

if $r$ is sufficiently large. Moreover, (3.13) certainly holds for every $r \in \mathbb{Z}^{+}$if $C_{i}=\mathbb{R}^{n}$ (so that $d\left(x^{\kappa_{r}}, C_{i}\right)=0$ ), by Lemma 2.5. It follows that, for every index $q$,

$$
\left\|x^{\kappa_{r+1}}-c\right\|^{2} \leq\left\|x^{\kappa_{r}}-c\right\|^{2}-\frac{f_{r}}{4} \max _{1 \leq i \leq q} d\left(x^{\kappa_{r}}, C_{i}\right)^{2}
$$

if $r$ is sufficiently large.

We claim that for any index $q$ there is a positive integer $k_{q}$ such that:

$$
\max _{1 \leq i \leq q} d\left(x^{k_{q}}, C_{i}\right) \leq 1 / q
$$

Indeed, if for some index $q$ no such $k_{q}$ existed then it would follow from (3.14) that

$$
\left\|x^{\kappa_{r+1}}-c\right\|^{2} \leq\left\|x^{\kappa_{r}}-c\right\|^{2}-\frac{f_{r}}{4 q^{2}}
$$

holds for all sufficiently large $r$, which is impossible as $\sum_{r=0}^{\infty} f_{r}$ diverges.

The existence for each index $q$ of some $k_{q}$ that satisfies (3.15) is enough to guarantee convergence of $x^{0}, x^{1}, x^{2}, \ldots$ to a point in $\bigcap \mathcal{C}$, as we observed in Claim 3.4.

\section{Third Convergence Theorem}

We continue to assume that $x^{0}, x^{1}, x^{2}, x^{3}, \ldots$ is an arbitrary sequence of points in $\mathbb{R}^{n}$ that satisfy (1.1) for some weight functions $w^{0}, w^{1}, w^{2}, w^{3}, \cdots: \mathbb{I} \rightarrow[0,1]$. The convergence theorem of this section is similar in form to the First Convergence Theorem. The difference is that, instead of assuming $\bigcap \mathcal{C}$ is an $n$-dimensional subset of $\mathbb{R}^{n}$, it assumes $\operatorname{UASC}(\mathcal{C})$ holds:

Theorem 4.1 (Third Convergence Theorem). Suppose UASC $(\mathcal{C})$ holds. Then the sequence $x^{0}, x^{1}, x^{2}, x^{3}, \ldots$ converges, and its limit lies in $C_{j}$ for every index $j$ such that $\sum_{k=0}^{\infty} \sum_{\iota \in \mathbb{I}\langle j\rangle} w^{k}(\iota)=\infty$. In particular, the sequence $x^{0}, x^{1}, x^{2}, \ldots$ converges to a point in $\bigcap \mathcal{C}$ if $\sum_{k=0}^{\infty} \sum_{\iota \in \mathbb{I}\langle j\rangle} w^{k}(\iota)=\infty$ for every index $j$ such that $C_{j} \neq \mathbb{R}^{n}$.

This theorem will be proved in the rest of this section. 


\subsection{Useful Lemmas}

Our proof of the Third Convergence Theorems will depend on the lemmas in this subsection. We note that these lemmas are true regardless of whether or not $\mathbf{U A S C}(\mathcal{C})$ holds.

For any point $s \in \mathbb{R}^{n}$, let $\mathrm{I}(s)$ and $\overline{\mathrm{I}}(s)$ be the complementary sets of indices and $\mathbb{I}(s)$ and $\overline{\mathbb{I}}(s)$ the complementary sets of index vectors that are defined as follows:

$$
\begin{aligned}
& \mathrm{I}(s)=\left\{i \in \mathbb{Z}^{+} \mid s \in C_{i}\right\} \\
& \overline{\mathrm{I}}(s)=\left\{i \in \mathbb{Z}^{+} \mid s \notin C_{i}\right\}=\mathbb{Z}^{+} \backslash \mathrm{I}(s) \\
& \mathbb{I}(s)=\{\iota \in \mathbb{I} \mid \operatorname{IndexSet}(\iota) \subseteq \mathrm{I}(s)\} \\
& \overline{\mathbb{I}}(s)=\{\iota \in \mathbb{I} \mid \operatorname{IndexSet}(\iota) \cap \overline{\mathrm{I}}(s) \neq \emptyset\}=\bigcup_{i \in \overline{\mathrm{I}}(s)} \mathbb{I}\langle i\rangle=\mathbb{I} \backslash \mathbb{I}(s)
\end{aligned}
$$

If $s \notin \bigcap \mathcal{C}$, then $\overline{\mathrm{I}}(s)$ and $\overline{\mathbb{I}}(s)$ are nonempty; if $s \in \bigcap \mathcal{C}$, then $\overline{\mathrm{I}}(s)$ and $\overline{\mathbb{I}}(s)$ are empty.

As $\iota \in \mathbb{I}(s)$ if and only if $s \in \bigcap_{i \in \operatorname{IndexSet}(\iota)} C_{i}$, assertion 1 of Lemma 2.4 implies:

Lemma 4.2. Let $s, z \in \mathbb{R}^{n}$ and let $\iota \in \mathbb{I}(s)$. Then $\|P[\iota](z)-s\| \leq\|z-s\|$.

The number $2 d\left(x^{0}, \cap \mathcal{C}\right)$ will be denoted by $M$. Our next lemma gives an upper bound (in terms of $M$ ) on how fast the iterates $x^{k}$ can move away from any given point $s \in \mathbb{R}^{n}$ as the iteration number $k$ increases. Note that $M$ does not depend on the iteration numbers $r_{1}$ and $r_{2}$ of this lemma.

Lemma 4.3. Let $r_{1}$ and $r_{2}$ be iteration numbers such that $r_{1}<r_{2}$, and let $s \in \mathbb{R}^{n}$. Then we have that $\left\|x^{r_{2}}-s\right\| \leq\left\|x^{r_{1}}-s\right\|+M \sum_{k=r_{1}}^{r_{2}-1} \sum_{\iota \in \overline{\mathbb{I}}(s)} w^{k}(\iota)$.

Proof. For all index vectors $\iota$, all iteration numbers $k$, and every $c \in \bigcap \mathcal{C}$, we see from Lemmas 2.4 and 2.5 that $\left\|P[\iota]\left(x^{k}\right)-c\right\| \leq\left\|x^{k}-c\right\| \leq\left\|x^{0}-c\right\|$, which (by the triangle inequality) implies $\left\|P[\iota]\left(x^{k}\right)-x^{k}\right\| \leq 2\left\|x^{0}-c\right\|$. Since this is true for every point $c$ in $\bigcap \mathcal{C}$, we have that

$$
\left\|P[\iota]\left(x^{k}\right)-x^{k}\right\| \leq 2 d\left(x^{0}, \cap \mathcal{C}\right)=M \quad \text { for all } k \geq 0
$$

and hence that

$$
\left\|P[\iota]\left(x^{k}\right)-s\right\| \leq\left\|x^{k}-s\right\|+\left\|P[\iota]\left(x^{k}\right)-x^{k}\right\| \leq\left\|x^{k}-s\right\|+M \quad \text { for all } k \geq 0 .
$$

Moreover, if $\iota \in \mathbb{I}(s)$ then Lemma 4.2 implies that

$$
\left\|P[\iota]\left(x^{k}\right)-s\right\| \leq\left\|x^{k}-s\right\| \quad \text { for all } k \geq 0 .
$$

Hence

$$
\begin{array}{rlrl}
\left\|x^{k+1}-s\right\| & =\left\|\left(\sum_{\iota \in \mathbb{I}} w^{k}(\iota) P[\iota]\left(x^{k}\right)\right)-s\right\| & \\
\leq & \sum_{\iota \in \mathbb{I}} w^{k}(\iota)\left\|P[\iota]\left(x^{k}\right)-s\right\| & & \text { by convexity of } u \mapsto\|u-s\| \\
& =\sum_{\iota \in \mathbb{I}(s)} w^{k}(\iota)\left\|P[\iota]\left(x^{k}\right)-s\right\| & & \\
& \quad+\sum_{\iota \in \bar{I}(s)} w^{k}(\iota)\left\|P[\iota]\left(x^{k}\right)-s\right\| & & \\
\leq & \sum_{\iota \in \mathbb{I}(s)} w^{k}(\iota)\left\|x^{k}-s\right\| & & \\
& \quad+\sum_{\iota \in \mathbb{I}(s)} w^{k}(\iota)\left(\left\|x^{k}-s\right\|+M\right) & & \text { by (4.2) and (4.1) } \\
& =\left\|x^{k}-s\right\|+M \sum_{\iota \in \mathbb{I}(s)} w^{k}(\iota) & & \text { as } \sum_{\iota \in \mathbb{I}} w^{k}(\iota)=1
\end{array}
$$

for all iteration numbers $k$. The lemma follows from this.

Corollary 4.4. Let $s$ be the limit of a convergent subsequence of $x^{0}, x^{1}, x^{2}, \ldots$, and suppose the series $\sum_{k=0}^{\infty} \sum_{\iota \in \overline{\mathbb{I}}(s)} w^{k}(\iota)$ converges. Then the sequence $x^{0}, x^{1}, x^{2}, \ldots$ converges to $s$. 
Proof. For all $\epsilon>0$, let $N(\epsilon)$ be an iteration number such that $M \sum_{r=N(\epsilon)}^{\infty} \sum_{\iota \in \overline{\mathbb{I}}(s)} w^{r}(\iota)<\epsilon / 2$, and let $\kappa(\epsilon)$ be an iteration number such that $\kappa(\epsilon) \geq N(\epsilon)$ and $\left\|x^{\kappa(\epsilon)}-s\right\|<\epsilon / 2$. Then, for all $\epsilon>0$, we see from Lemma 4.3 that $\left\|x^{k}-s\right\|<\epsilon$ when $k \geq \kappa(\epsilon)$.

For all $s \in \mathbb{R}^{n} \backslash \bigcap \mathcal{C}$, let us now define:

$$
d(s)=d\left(s, \bigcup_{i \in \overline{\mathrm{I}}(s)} C_{i}\right)
$$

Lemma 4.5. Let $c \in \bigcap \mathcal{C}$, let $s \in \mathbb{R}^{n} \backslash \bigcap \mathcal{C}$, let $\iota \in \overline{\mathbb{I}}(s)$, and let $z$ be a point in $\mathbb{R}^{n}$ such that $\|z-s\| \leq d(s) / 2$. Then we have that $\|P[\iota](z)-c\|^{2} \leq\|z-c\|^{2}-d(s)^{2} / 4$.

Proof. Let $\iota=\left(\iota_{1}, \ldots, \iota_{m}\right)$, so that $P[\iota]=P_{\iota_{m}} \circ \ldots \circ P_{\iota_{1}}$. Let $\iota_{j}$ be the first one of the components $\iota_{1}, \ldots, \iota_{m}$ for which $s \notin C_{\iota_{j}} ; \iota_{j}$ exists, since $\iota \in \overline{\mathbb{I}}(s)$. Let $\iota^{\prime}=\left(\iota_{1}, \ldots, \iota_{j-1}\right)$ and let $\iota^{\prime \prime}=\left(\iota_{j+1}, \ldots, \iota_{m}\right)$, so that $P[\iota]=P\left[\iota^{\prime \prime}\right] \circ P_{\iota_{j}} \circ P\left[\iota^{\prime}\right]$. (If $j=1$ or $j=m$, then $\iota^{\prime}$ or $\iota^{\prime \prime}$ is the empty sequence and we define $P\left[\iota^{\prime}\right]$ or $P\left[\iota^{\prime \prime}\right]$ to be the identity map.) As $\iota^{\prime} \in \mathbb{I}(s)$ if $j \neq 1$, we see from Lemma 4.2 that $\left\|P\left[\iota^{\prime}\right](z)-s\right\| \leq\|z-s\| \leq d(s) / 2$. So, since $d(s)=d\left(s, \bigcup_{i \in \overline{\mathrm{I}}(s)} C_{i}\right)$, it follows from the triangle inequality that the point $P\left[\iota^{\prime}\right](z)$ satisfies:

$$
d\left(P\left[\iota^{\prime}\right](z), \bigcup_{i \in \overline{\mathrm{I}}(s)} C_{i}\right) \geq d(s)-d(s) / 2=d(s) / 2
$$

From this it follows that

$$
\begin{array}{rlrl}
\|P[\iota](z)-c\|^{2} & =\left\|P\left[\iota^{\prime \prime}\right]\left(P_{\iota_{j}}\left(P\left[\iota^{\prime}\right](z)\right)\right)-c\right\|^{2} & \\
& \leq\left\|P_{\iota_{j}}\left(P\left[\iota^{\prime}\right](z)\right)-c\right\|^{2} & & \text { by Lemma 2.4 } \\
& \leq\left\|P\left[\iota^{\prime}\right](z)-c\right\|^{2}-d\left(P\left[\iota^{\prime}\right](z), C_{\iota_{j}}\right)^{2} & & \text { by Proposition 2.3 } \\
& \leq\|z-c\|^{2}-d\left(P\left[\iota^{\prime}\right](z), C_{\iota_{j}}\right)^{2} & & \text { by Lemma 2.4 } \\
& \leq\|z-c\|^{2}-d\left(P\left[\iota^{\prime}\right](z), \bigcup_{i \in \overline{\mathrm{I}}(s)} C_{i}\right)^{2} & & \text { since } \iota_{j} \in \overline{\mathrm{I}}(s) \\
& \leq\|z-c\|^{2}-d(s)^{2} / 4 & & \text { by 44.31) }
\end{array}
$$

and so the proof is complete.

Lemma 4.6. Let $c \in \bigcap \mathcal{C}$ and let $s \in \mathbb{R}^{n} \backslash \bigcap \mathcal{C}$. Let $k$ be any iteration number such that $\left\|x^{k}-s\right\| \leq d(s) / 2$. Then we have that $\left\|x^{k+1}-c\right\|^{2} \leq\left\|x^{k}-c\right\|^{2}-d(s)^{2} \sum_{\iota \in \bar{I}(s)} w^{k}(\iota) / 4$.

Proof. We have that

$$
\begin{aligned}
& \left\|x^{k+1}-c\right\|^{2} \leq \sum_{\iota \in \overline{\mathbb{I}}(s)} w^{k}(\iota)\left\|P[\iota]\left(x^{k}\right)-c\right\|^{2} \\
& +\quad \sum_{\iota \in \mathbb{I}(s)} w^{k}(\iota)\left\|P[\iota]\left(x^{k}\right)-c\right\|^{2} \quad \text { by (2.6) } \\
& \leq \sum_{\iota \in \overline{\mathbb{I}}(s)} w^{k}(\iota)\left(\left\|x^{k}-c\right\|^{2}-d(s)^{2} / 4\right) \\
& +\quad \sum_{\iota \in \mathbb{I}(s)} w^{k}(\iota)\left\|x^{k}-c\right\|^{2} \quad \text { by Lemmas } 4.5 \text { and } 2.4 \\
& =\left\|x^{k}-c\right\|^{2}-\sum_{\iota \in \overline{\mathbb{I}}(s)} w^{k}(\iota) d(s)^{2} / 4 \quad \text { since } \sum_{\iota \in \mathbb{I}} w^{k}(\iota)=1
\end{aligned}
$$

as required.

Corollary 4.7. Let $c \in \cap \mathcal{C}$, let $s \in \mathbb{R}^{n} \backslash \cap \mathcal{C}$, and let $r_{1}<r_{2}$ be iteration numbers such that:

$$
\left\|x^{k}-s\right\| \leq d(s) / 2 \quad \text { for each } k \text { in the range } r_{1} \leq k \leq r_{2}-1 \text {. }
$$

Then we have that $\left\|x^{r_{2}}-c\right\|^{2} \leq\left\|x^{r_{1}}-c\right\|^{2}-d(s)^{2} \sum_{k=r_{1}}^{r_{2}-1} \sum_{\iota \in \overline{\mathbb{I}}(s)} w^{k}(\iota) / 4$.

From this corollary and Lemma 4.3 we now deduce: 
Lemma 4.8. Suppose $x_{0} \notin \bigcap \mathcal{C}$, so that $M>0$. Let $c \in \bigcap \mathcal{C}$, let $s \in \mathbb{R}^{n} \backslash \bigcap \mathcal{C}$, and let $r_{1}$ and $r_{2}$ be iteration numbers such that $r_{1}<r_{2}$ for which the following conditions hold:

1. $\left\|x^{r_{1}}-s\right\| \leq d(s) / 4$.

2. $\sum_{k=r_{1}}^{r_{2}-2} \sum_{\iota \in \bar{I}(s)} w^{k}(\iota) \leq d(s) / 4 M$.

Then $\left\|x^{r_{2}}-c\right\|^{2} \leq\left\|x^{r_{1}}-c\right\|^{2}-d(s)^{2} \sum_{k=r_{1}}^{r_{2}-1} \sum_{\iota \in \bar{I}(s)} w^{k}(\iota) / 4$.

Proof. In view of Corollary 4.7, it is enough to show $\left\|x^{k}-s\right\| \leq d(s) / 2$ for each $k$ in the range $r_{1} \leq k \leq r_{2}-1$. This is indeed the case, because for any such $k$ we have that

$$
\begin{array}{rlrl}
\left\|x^{k}-s\right\| & \leq\left\|x^{r_{1}}-s\right\|+M \sum_{j=r_{1}}^{k-1} \sum_{\iota \in \overline{\mathbb{I}}(s)} w^{k}(\iota) & \text { by Lemma 4.3 } \\
& \leq\left\|x^{r_{1}}-s\right\|+M \sum_{j=r_{1}}^{r_{2}-2} \sum_{\iota \in \mathbb{I}(s)} w^{k}(\iota) & \\
& \leq d(s) / 4+d(s) / 4=d(s) / 2 & & \text { by conditions } 1 \text { and } 2
\end{array}
$$

as required.

If $\sum_{k=0}^{\infty} \sum_{\iota \in \overline{\mathbb{I}}(s)} w^{k}(\iota)$ diverges then, for any $r_{1}$ that satisfies condition 1 of Lemma 4.8 , how can we choose $r_{2}$ to get the best lower bound for $\left\|x^{r_{1}}-c\right\|^{2}-\left\|x^{r_{2}}-c\right\|^{2}$ from this lemma? The answer is that we should choose $r_{2}$ to be the greatest value for which condition 2 of Lemma 4.8 is satisfied. Most importantly, for that choice of $r_{2}$ we get a lower bound for the difference $\left\|x^{r_{1}}-c\right\|^{2}-\left\|x^{r_{2}}-c\right\|^{2}$ which does not depend on $r_{1}$. Thinking along these lines, we deduce the following corollary of the lemma:

Corollary 4.9. Suppose $x_{0} \notin \bigcap \mathcal{C}$, so that $M>0$. Let $c \in \cap \mathcal{C}$, and let $s \in \mathbb{R}^{n} \backslash \cap \mathcal{C}$ be such that the series $\sum_{k=0}^{\infty} \sum_{\iota \in \mathbb{I}(s)} w^{k}(\iota)$ diverges. Then for any iteration number $\kappa$ such that

$$
\left\|x^{\kappa}-s\right\| \leq d(s) / 4
$$

the following inequality holds for all sufficiently large iteration numbers $\kappa^{\prime}$ :

$$
\left\|x^{\kappa^{\prime}}-c\right\|^{2} \leq\left\|x^{\kappa}-c\right\|^{2}-d(s)^{3} / 16 M
$$

Proof. Let $\kappa$ be an iteration number for which (4.4) holds, let $r_{1}=\kappa$, and let $r_{2}$ be the iteration number such that $r_{2} \geq r_{1}+1$ and both of the following are true:

$$
\begin{aligned}
& \sum_{k=r_{1}}^{r_{2}-2} \sum_{\iota \in \overline{\mathbb{I}}(s)} w^{k}(\iota) \leq d(s) / 4 M \\
& \sum_{k=r_{1}}^{r_{2}-1} \sum_{\iota \in \overline{\mathbb{I}}(s)} w^{k}(\iota)>d(s) / 4 M
\end{aligned}
$$

Note that $r_{2}$ must exist, since the series $\sum_{k=0}^{\infty} \sum_{\iota \in \mathbb{I}(s)} w^{k}(\iota)$ diverges. It now follows from (4.4) and (4.6) that the conditions of Lemma 4.8 hold. From this, (4.7), and the conclusion of Lemma 4.8 we see that (4.5) holds for $\kappa^{\prime}=r_{2}$, whence (4.5) also holds for all $\kappa^{\prime}>r_{2}$ (by Lemma 2.5).

Corollary 4.10. Suppose $x_{0} \notin \bigcap \mathcal{C}$, so that $M>0$. Let $s \in \mathbb{R}^{n} \backslash \bigcap \mathcal{C}$ be such that $d(s)=d\left(s, \bigcup_{i \in \bar{I}(s)} C_{i}\right)>0$ and the series $\sum_{k=0}^{\infty} \sum_{\iota \in \mathbb{I}(s)} w^{k}(\iota)$ diverges. Then there are at most finitely many iteration numbers $k$ such that $\left\|x^{k}-s\right\| \leq d(s) / 4$.

Proof. Suppose for contradiction that $\left\|x^{k}-s\right\| \leq d(s) / 4$ for infinitely many iteration numbers $k$. Let $c$ be any point in $\bigcap \mathcal{C}$. Then we see from Corollary 4.9 that for every iteration number $k$ such that $\left\|x^{k}-s\right\| \leq d(s) / 4$ there exists an iteration number $k^{\prime}>k$ such that both of the following are true:

$$
\begin{gathered}
\left\|x^{k^{\prime}}-s\right\| \leq d(s) / 4 \\
\left\|x^{k^{\prime}}-c\right\|^{2} \leq\left\|x^{k}-c\right\|^{2}-d(s)^{3} / 16 M
\end{gathered}
$$


Hence there is an infinite sequence $k_{0}<k_{1}<k_{2}<\ldots$ such that the following are true for every $j \geq 1$ :

$$
\begin{gathered}
\left\|x^{k_{j}}-s\right\| \leq d(s) / 4 \\
\left\|x^{k_{j}}-c\right\|^{2} \leq\left\|x^{k_{j-1}}-c\right\|^{2}-d(s)^{3} / 16 M
\end{gathered}
$$

But (4.8) cannot hold for all $j \geq 1$ because $d(s)^{3} / 16 M$ is a positive value that is the same for every $j$ and $\left\|x^{k_{j}}-c\right\|^{2}$ is nonnegative for all $j$.

\subsection{Proof of the Third Convergence Theorem}

We see from Lemma 2.5 that $x^{0}, x^{1}, x^{2}, \ldots$ is a bounded sequence in $\mathbb{R}^{n}$, and must therefore have a convergent subsequence. Let $s$ be the limit of a convergent subsequence of $x^{0}, x^{1}, x^{2}, \ldots$

If $s \in \bigcap \mathcal{C}$, then it follows from Lemma 3.3 that $x^{0}, x^{1}, x^{2}, \ldots$ converges to $s$ and the Third Convergence Theorem holds.

So let us now assume $s \notin \cap \mathcal{C}$. Under the hypotheses of the theorem, $\operatorname{UASC}(\mathcal{C})$ holds and therefore $d(s)>0$. As $s$ is the limit of a convergent subsequence of $x^{0}, x^{1}, x^{2}, \ldots$ and $d(s)>0$, it follows from Corollary 4.10 that the series $\sum_{k=0}^{\infty} \sum_{\iota \in \mathbb{I}(s)} w^{k}(\iota)$ cannot diverge. So we see from Corollary 4.4 that the sequence $x^{0}, x^{1}, x^{2}, \ldots$ converges to $s$.

Since $\overline{\mathbb{I}}(s) \supseteq \mathbb{I}\langle i\rangle$ for every index $i$ in $\overline{\mathrm{I}}(s)$, the fact that the series $\sum_{k=0}^{\infty} \sum_{\iota \in \overline{\mathbb{I}}(s)} w^{k}(\iota)$ does not diverge implies there is no $i \in \overline{\mathrm{I}}(s)$ for which the series $\sum_{k=0}^{\infty} \sum_{\iota \in \mathbb{I}\langle i\rangle} w^{k}(\iota)$ diverges. Equivalently, there is no index $i$ such that $s \notin C_{i}$ for which the series $\sum_{k=0}^{\infty} \sum_{\iota \in \mathbb{I}\langle i\rangle} w^{k}(\iota)$ diverges.

\section{Fourth Convergence Theorem}

We now consider perturbed versions of sequences that satisfy equation (1.1). For each iteration number $k$, let $w^{k}: \mathbb{I} \rightarrow[0,1]$ be a weight function, let $\boldsymbol{v}^{k}$ be a vector in $\mathbb{R}^{n}$, and let $T_{k}: \mathbb{R}^{n} \rightarrow \mathbb{R}^{n}$ be defined by

$$
T_{k}(x)=\sum_{\iota \in \mathbb{I}} w^{k}(\iota) P[\iota](x) \quad \text { for all } x \in \mathbb{R}^{n} .
$$

Let $x_{*}^{0}$ be an arbitrary point in $\mathbb{R}^{n}$, and let $x_{*}^{1}, x_{*}^{2}, x_{*}^{3}, \cdots \in \mathbb{R}^{n}$ be defined by

$$
x_{*}^{k+1}=T_{k}\left(x_{*}^{k}\right)+\boldsymbol{v}^{k}=\left(\sum_{\iota \in \mathbb{I}} w^{k}(\iota) P[\iota]\left(x_{*}^{k}\right)\right)+\boldsymbol{v}^{k} \quad \text { for every } k \geq 0 .
$$

Our next convergence theorem says that, if the series $\sum_{i=0}^{\infty}\left\|\boldsymbol{v}^{k}\right\|$ converges, then our three earlier convergence theorems remain valid if we substitute $x_{*}^{0}, x_{*}^{1}, x_{*}^{2}, x_{*}^{3}, \ldots$ for $x^{0}, x^{1}, x^{2}, x^{3}, \ldots$ :

Theorem 5.1 (Fourth Convergence Theorem). Suppose the series $\sum_{k=0}^{\infty}\left\|\boldsymbol{v}^{k}\right\|$ converges. Then:

1. If $\bigcap \mathcal{C}$ is an $n$-dimensional subset of $\mathbb{R}^{n}$ or $\mathbf{U A S C}(\mathcal{C})$ holds, then the sequence $x_{*}^{0}, x_{*}^{1}, x_{*}^{2}, \ldots$ converges, and its limit lies in $C_{j}$ for every index $j$ such that $\sum_{k=0}^{\infty} \sum_{\iota \in \mathbb{I}\langle j\rangle} w^{k}(\iota)=\infty$. In particular, if $\cap \mathcal{C}$ is an n-dimensional subset of $\mathbb{R}^{n}$ or $\mathbf{U A S C}(\mathcal{C})$ holds, and $\sum_{k=0}^{\infty} \sum_{\iota \in \mathbb{I}\langle j\rangle} w^{k}(\iota)=\infty$ for every index $j$ such that $C_{j} \neq \mathbb{R}^{n}$, then $x_{*}^{0}, x_{*}^{1}, x_{*}^{2}, \ldots$ converges to a point in $\bigcap \mathcal{C}$.

2. If the weight functions $w^{0}, w^{1}, w^{2}, \ldots$ satisfy conditions $\boldsymbol{C 1}$ and $\boldsymbol{C 2}$ of the Second Convergence Theorem for some $f_{1}, f_{2}, f_{3}, \cdots \in[0,1]$ such that $\sum_{r=1}^{\infty} f_{r}=\infty$ and a sequence of iteration numbers $\kappa_{1}<\kappa_{2}<\kappa_{3}<\ldots$, then the sequence $x_{*}^{0}, x_{*}^{1}, x_{*}^{2}, \ldots$ converges to a point in $\bigcap \mathcal{C}$.

Important examples of iterates satisfying (5.1) for perturbation vectors $\left(\boldsymbol{v}^{k}\right)_{k=0}^{\infty}$ such that $\sum_{k=0}^{\infty}\left\|\boldsymbol{v}^{k}\right\|$ converges are the sequences $\left(x_{* *}^{k}\right)_{k=0}^{\infty}$ defined by

$$
x_{* *}^{k+1}=T_{k}\left(x_{* *}^{k}+\beta_{k} \boldsymbol{u}^{k}\right)=\sum_{\iota \in \mathbb{I}} w^{k}(\iota) P[\iota]\left(x_{* *}^{k}+\beta_{k} \boldsymbol{u}^{k}\right) \quad \text { for every } k \geq 0
$$

where $\left(\boldsymbol{u}^{k}\right)_{k=0}^{\infty}$ may be any bounded sequence of vectors and $\left(\beta_{k}\right)_{k=0}^{\infty}$ any sequence of scalars such that $\sum_{k=0}^{\infty}\left|\beta_{k}\right|<\infty$ : Indeed, if (5.2) holds and we define $\boldsymbol{v}^{k}=T_{k}\left(x_{* *}^{k}+\beta_{k} \boldsymbol{u}^{k}\right)-T_{k}\left(x_{* *}^{k}\right)$, then (5.1) holds with $\left(x_{* *}^{k}\right)_{k=0}^{\infty}$ in place of $\left(x_{*}^{k}\right)_{k=0}^{\infty}$ and it will follow from Lemma 5.5 below that $\left\|\boldsymbol{v}^{k}\right\| \leq\left\|\beta_{k} \boldsymbol{u}^{k}\right\|$ for every $k$ 
(whence $\sum_{k=0}^{\infty}\left\|\boldsymbol{v}^{k}\right\|$ converges). The superiorization methodology Cens15, CHJ17, derives iterative schemes of the form (5.2) from iterative schemes of the form (1.1) by choosing vectors $\beta_{k} \boldsymbol{u}^{k}$ that steer the sequence of iterates with the aim of improving the efficacy of the iterative scheme.

The Fourth Convergence Theorem is an immediate consequence of statements 1 and 3 of Proposition 5.6 below. Our proof of that proposition will depend on the next three lemmas. We will give a self-contained argument that deduces Proposition 5.6 (and hence our Fourth Convergence Theorem) from these lemmas. But when $\sum_{k=0}^{\infty} \sum_{\iota \in \mathbb{I}\langle j\rangle} w^{k}(\iota)=\infty$ for every index $j$ such that $C_{j} \neq \mathbb{R}^{n}$ (which is always true under the hypotheses of statement 2 of the theorem) the theorem follows at once from these lemmas and a result of Butnariu, Reich, and Zaslavski BuRZ08, Thm. 3.2], while Proposition [5.6 follows at once from the lemmas and BaRZ18, Thm. 11].

For all pairs of nonnegative integers $(k, l)$ such that $k \geq l$, we define the point $x_{l}^{k} \in \mathbb{R}^{n}$ as follows:

$$
\begin{aligned}
x_{0}^{0} & =x_{*}^{0} & & \\
x_{l}^{k+1} & =T_{k}\left(x_{l}^{k}\right) & & \text { whenever } k \geq l \geq 0 . \\
x_{k+1}^{k+1} & =x_{k}^{k+1}+\boldsymbol{v}^{k} & & \text { for all } k \geq 0 .
\end{aligned}
$$

From (5.5) and the case $l=k$ of (5.4) we see that $x_{k+1}^{k+1}=T_{k}\left(x_{k}^{k}\right)+\boldsymbol{v}^{k}$ for all $k \geq 0$. It follows from this, (5.3), and (5.1), that:

$$
x_{k}^{k}=x_{*}^{k} \quad \text { for all } k \geq 0 .
$$

Figure 5.1 shows how different points $x_{l}^{k}$ relate to each other.

Lemma 5.2. Suppose at least one of the following is true:

1. $\cap \mathcal{C}$ is an $n$-dimensional subset of $\mathbb{R}^{n}$.

2. $\mathbf{U A S C}(\mathcal{C})$ holds.

Then for every nonnegative integer $l$ the sequence $\left(\left(T_{l+k} \circ \cdots \circ T_{l}\right)\left(x_{l}^{l}\right)\right)_{k=0}^{\infty}=x_{l}^{l+1}, x_{l}^{l+2}, x_{l}^{l+3}, \ldots$ converges, and its limit lies in $C_{j}$ for every index $j$ such that $\sum_{k=0}^{\infty} \sum_{\iota \in \mathbb{I}\langle j\rangle} w^{k}(\iota)=\infty$.

Proof. Let $l$ be any nonnegative integer. We see from Figure 5.1 that equation (1.1) holds when we substitute $x_{l}^{l}, x_{l}^{l+1}, x_{l}^{l+2}, x_{l}^{l+3}, \ldots$ and $w^{l}, w^{l+1}, w^{l+2}, w^{l+3}, \ldots$ for $x^{0}, x^{1}, x^{2}, x^{3}, \ldots$ and $w^{0}, w^{1}, w^{2}, w^{3}, \ldots$ So it follows from the First and the Third Convergence Theorems that the sequence $x_{l}^{l}, x_{l}^{l+1}, x_{l}^{l+2}, x_{l}^{l+3}, \ldots$ converges, and that its limit lies in $C_{j}$ for every index $j$ such that $\sum_{k=0}^{\infty} \sum_{\iota \in \mathbb{I}\langle j\rangle} w^{l+k}(\iota)=\infty$. As $\sum_{k=0}^{\infty} \sum_{\iota \in \mathbb{I}\langle j\rangle} w^{l+k}(\iota)=\infty$ just if $\sum_{k=0}^{\infty} \sum_{\iota \in \mathbb{I}\langle j\rangle} w^{k}(\iota)=\infty$, the lemma follows.

Lemma 5.3. Suppose the sequence of weight functions $\left(w^{k}\right)_{k=0}^{\infty}$ satisfies conditions $\boldsymbol{C 1}$ and $\boldsymbol{C} 2$ of the Second Convergence Theorem for some sequence $\left(f_{r}\right)_{r=1}^{\infty}$ of numbers in $[0,1]$ such that $\sum_{r=1}^{\infty} f_{r}=\infty$ and some strictly increasing sequence of iteration numbers $\left(\kappa_{r}\right)_{r=1}^{\infty}$. Then for every nonnegative integer $l$ the sequence $\left(\left(T_{l+k} \circ \cdots \circ T_{l}\right)\left(x_{l}^{l}\right)\right)_{k=0}^{\infty}=x_{l}^{l+1}, x_{l}^{l+2}, x_{l}^{l+3}, \ldots$ converges to a point in $\bigcap \mathcal{C}$.

Proof. Let $l$ be any nonnegative integer, and $\rho(l)$ any nonnegative integer such that $\kappa_{\rho(l)+1} \geq l$. For all $r \in \mathbb{Z}^{+}$, we will write $f_{r}^{\langle l\rangle}$ for $f_{\rho(l)+r}$ and write $\kappa_{r}^{\langle l\rangle}$ for $\kappa_{\rho(l)+r}-l$. Note that $\left(f_{r}^{\langle l\rangle}\right)_{r=1}^{\infty}$ is a sequence of numbers in $[0,1]$ such that $\sum_{r=1}^{\infty} f_{r}^{\langle l\rangle}=\infty$. Moreover, we see from the definition of $\rho(l)$ that $\left(\kappa_{r}^{\langle l\rangle}\right)_{r=1}^{\infty}$ is a strictly increasing sequence of nonnegative integers (i.e., iteration numbers).

Claim. The sequence $\left(w^{l+k}\right)_{k=0}^{\infty}$ satisfies conditions $\mathbf{C} 1$ and $\mathbf{C 2}$ of the Second Convergence Theorem for the sequence $\left(f_{r}^{\langle l\rangle}\right)_{r=1}^{\infty}$ and the sequence of iteration numbers $\left(\kappa_{r}^{\langle l\rangle}\right)_{r=1}^{\infty}$. 


$$
\begin{aligned}
& x_{0}^{0} \stackrel{T_{0}}{\longmapsto} x_{0}^{1} \stackrel{T_{1}}{\longmapsto} x_{0}^{2} \stackrel{T_{2}}{\longmapsto} x_{0}^{3} \stackrel{T_{3}}{\longmapsto} x_{0}^{4} \stackrel{T_{4}}{\longmapsto} x_{0}^{5} \stackrel{T_{5}}{\longmapsto} x_{0}^{6} \stackrel{T_{6}}{\longmapsto} x_{0}^{7} \stackrel{T_{7}}{\longmapsto} x_{0}^{8} \stackrel{T_{8}}{\longmapsto} \ldots x_{0}^{\infty} \\
& \downarrow+\boldsymbol{v}^{0} \\
& x_{1}^{1} \stackrel{T_{1}}{\longmapsto} x_{1}^{2} \stackrel{T_{2}}{\longmapsto} x_{1}^{3} \stackrel{T_{3}}{\longmapsto} x_{1}^{4} \stackrel{T_{4}}{\longmapsto} x_{1}^{5} \stackrel{T_{5}}{\longmapsto} x_{1}^{6} \stackrel{T_{6}}{\longmapsto} x_{1}^{7} \stackrel{T_{7}}{\longmapsto} x_{1}^{8} \stackrel{T_{8}}{\longmapsto} \ldots x_{1}^{\infty} \\
& \downarrow+\boldsymbol{v}^{1} \\
& x_{2}^{2} \stackrel{T_{2}}{\longmapsto} x_{2}^{3} \stackrel{T_{3}}{\longmapsto} x_{2}^{4} \stackrel{T_{4}}{\longmapsto} x_{2}^{5} \stackrel{T_{5}}{\longmapsto} x_{2}^{6} \stackrel{T_{6}}{\longmapsto} x_{2}^{7} \stackrel{T_{7}}{\longmapsto} x_{2}^{8} \stackrel{T_{8}}{\longmapsto} \ldots x_{2}^{\infty} \\
& \downarrow+v^{2} \\
& x_{3}^{3} \stackrel{T_{3}}{\longmapsto} x_{3}^{4} \stackrel{T_{4}}{\longmapsto} x_{3}^{5} \stackrel{T_{5}}{\longmapsto} x_{3}^{6} \stackrel{T_{6}}{\longmapsto} x_{3}^{7} \stackrel{T_{7}}{\longmapsto} x_{3}^{8} \stackrel{T_{8}}{\longmapsto} \ldots x_{3}^{\infty} \\
& \downarrow+v^{3} \\
& x_{4}^{4} \stackrel{T_{4}}{\longmapsto} x_{4}^{5} \stackrel{T_{5}}{\longmapsto} x_{4}^{6} \stackrel{T_{6}}{\longmapsto} x_{4}^{7} \stackrel{T_{7}}{\longmapsto} x_{4}^{8} \stackrel{T_{8}}{\longmapsto} \ldots x_{4}^{\infty} \\
& \downarrow+\boldsymbol{v}^{4} \\
& x_{5}^{5} \stackrel{T_{5}}{\longmapsto} x_{5}^{6} \stackrel{T_{6}}{\longmapsto} x_{5}^{7} \stackrel{T_{7}}{\longmapsto} x_{5}^{8} \stackrel{T_{8}}{\longmapsto} \ldots x_{5}^{\infty} \\
& \downarrow+v^{5} \\
& x_{6}^{6} \stackrel{T_{6}}{\longmapsto} x_{6}^{7} \stackrel{T_{7}}{\longmapsto} x_{6}^{8} \stackrel{T_{8}}{\longmapsto} \ldots x_{6}^{\infty}
\end{aligned}
$$

FiguRE 5.1. Relationships between different points $x_{l}^{k}$. Note that $x_{*}^{k}=x_{k}^{k}$ for all $k \geq 0$. Each point $x_{l}^{\infty}$ denotes the limit of the sequence $\left(x_{l}^{l+k}\right)_{k=0}^{\infty}$, which is convergent under the hypotheses of either part of the Fourth Convergence Theorem (by Lemmas 5.2 and [5.3).

As we observed in the proof of the previous lemma, equation (1.1) continues to hold when we substitute $w^{l}, w^{l+1}, w^{l+2}, w^{l+3}, \ldots$ and $x_{l}^{l}, x_{l}^{l+1}, x_{l}^{l+2}, x_{l}^{l+3}, \ldots$ for $w^{0}, w^{1}, w^{2}, w^{3}, \ldots$ and $x^{0}, x^{1}, x^{2}, x^{3}, \ldots$ So if the Claim is valid then it will follow from the Second Convergence Theorem that $x_{l}^{l}, x_{l}^{l+1}, x_{l}^{l+2}, x_{l}^{l+3}, \ldots$ converges to a point in $\bigcap \mathcal{C}$ and the lemma will be proved. To complete the proof, we proceed to justify the Claim.

For all $i, r \in \mathbb{Z}^{+}$, let $\mathbf{C} \mathbf{1}\left[\boldsymbol{w}_{0}, \boldsymbol{f}, \boldsymbol{\kappa}\right](r), \mathbf{C} \mathbf{1}\left[\boldsymbol{w}_{l}, \boldsymbol{f}^{\langle l\rangle}, \boldsymbol{\kappa}^{\langle l\rangle}\right](r), \mathbf{C 2}\left[\boldsymbol{w}_{0}, \boldsymbol{f}, \boldsymbol{\kappa}\right](i, r)$, and $\mathbf{C 2}\left[\boldsymbol{w}_{l}, \boldsymbol{f}^{\langle l\rangle}, \boldsymbol{\kappa}^{\langle l\rangle}\right](i, r)$ be the conditions defined as follows:

$$
\begin{array}{lll}
\mathbf{C 1}\left[\boldsymbol{w}_{0}, \boldsymbol{f}, \boldsymbol{\kappa}\right](r) & \Leftrightarrow & \sum_{k=\kappa_{r}}^{\kappa_{r+1}-2} \operatorname{MaxLength}\left(w^{k}\right) \leq \frac{1}{f_{r}} \text { if } f_{r}>0 \\
\mathbf{C} 1\left[\boldsymbol{w}_{l}, \boldsymbol{f}^{\langle l\rangle}, \boldsymbol{\kappa}^{\langle l\rangle}\right](r) & \Leftrightarrow \quad \sum_{k=\kappa_{r}^{\langle l\rangle}}^{\kappa_{r+1}^{\langle l}-2} \operatorname{MaxLength}\left(w^{l+k}\right) \leq \frac{1}{f_{r}^{\langle l\rangle}} \text { if } f_{r}^{\langle l\rangle}>0 \\
\mathbf{C 2}\left[\boldsymbol{w}_{0}, \boldsymbol{f}, \boldsymbol{\kappa}\right](i, r) & \Leftrightarrow \quad \max _{\kappa_{r} \leq k<\kappa_{r+1}} \sum_{\iota \in \mathbb{I}\langle i\rangle} \frac{w^{k}(\iota)}{\operatorname{Pos}(i, \iota)} \geq f_{r} \\
\mathbf{C 2}\left[\boldsymbol{w}_{l}, \boldsymbol{f}^{\langle l\rangle}, \boldsymbol{\kappa}^{\langle l\rangle}\right](i, r) \Leftrightarrow & \max _{\kappa_{r}^{\langle\langle\rangle} \leq k<\kappa_{r+1}^{\langle l\rangle}} \sum_{\iota \in \mathbb{I}\langle i\rangle} \frac{w^{l+k}(\iota)}{\operatorname{Pos}(i, \iota)} \geq f_{r}^{\langle l\rangle}
\end{array}
$$

Then the hypothesis that $\left(w^{k}\right)_{k=0}^{\infty}$ satisfies conditions $\mathbf{C} \mathbf{1}$ and $\mathbf{C} \mathbf{2}$ of the Second Convergence Theorem for the sequences $\left(f_{r}\right)_{r=1}^{\infty}$ and $\left(\kappa_{r}\right)_{r=1}^{\infty}$ is equivalent to the following assertions: 
1: $\mathbf{C 1}\left[\boldsymbol{w}_{0}, \boldsymbol{f}, \boldsymbol{\kappa}\right](r)$ holds for all $r \in \mathbb{Z}^{+}$.

2: For all $i \in \mathbb{Z}^{+}$such that $C_{i} \neq \mathbb{R}^{n}, \mathbf{C 2}\left[\boldsymbol{w}_{0}, \boldsymbol{f}, \boldsymbol{\kappa}\right](i, r)$ holds for all sufficiently large $r \in \mathbb{Z}^{+}$.

Assertions 1 and 2 imply the assertions obtained from them by replacing $\mathbf{C} \mathbf{1}\left[\boldsymbol{w}_{0}, \boldsymbol{f}, \boldsymbol{\kappa}\right](r)$ and $\mathbf{C 2}\left[\boldsymbol{w}_{0}, \boldsymbol{f}, \boldsymbol{\kappa}\right](i, r)$ with $\mathbf{C} \mathbf{1}\left[\boldsymbol{w}_{0}, \boldsymbol{f}, \boldsymbol{\kappa}\right](\rho(l)+r)$ and $\mathbf{C} 2\left[\boldsymbol{w}_{0}, \boldsymbol{f}, \boldsymbol{\kappa}\right](i, \rho(l)+r)$. The resulting assertions are equivalent to:

$1^{\prime}:$ For all $r \in \mathbb{Z}^{+}, \quad \sum_{k=\kappa_{\rho(l)+r}}^{\kappa_{\rho(l)+r+1}-2} \operatorname{MaxLength}\left(w^{k}\right) \leq \frac{1}{f_{\rho(l)+r}}$ if $f_{\rho(l)+r}>0$.

$2^{\prime}$ : For all $i \in \mathbb{Z}^{+}$such that $C_{i} \neq \mathbb{R}^{n}, \max _{\kappa_{\rho(l)+r} \leq k<\kappa_{\rho(l)+r+1}} \sum_{\iota \in \mathbb{I}\langle i\rangle} \frac{w^{k}(\iota)}{\operatorname{Pos}(i, \iota)} \geq f_{\rho(l)+r}$ for all sufficiently large $r \in \mathbb{Z}^{+}$.

Since the sequence $\left(w^{k}\right)_{\kappa_{\rho(l)+r} \leq k<\kappa_{\rho(l)+r+1}}$ is the same as $\left(w^{l+k}\right)_{\kappa_{r}^{\langle l\rangle} \leq k<\kappa_{r+1}^{\langle l\rangle}}$, and since $f_{\rho(l)+r}=f_{r}^{\langle l\rangle}$ for all $r \in \mathbb{Z}^{+}$, assertions $1^{\prime}$ and $2^{\prime}$ are respectively equivalent to:

$1^{\prime \prime}: \mathbf{C} \mathbf{1}\left[\boldsymbol{w}_{l}, \boldsymbol{f}^{\langle l\rangle}, \boldsymbol{\kappa}^{\langle l\rangle}\right](r)$ holds for all $r \in \mathbb{Z}^{+}$.

$2^{\prime \prime}$ : For all $i \in \mathbb{Z}^{+}$such that $C_{i} \neq \mathbb{R}^{n}, \mathbf{C 2}\left[\boldsymbol{w}_{l}, \boldsymbol{f}^{\langle l\rangle}, \boldsymbol{\kappa}^{\langle l\rangle}\right](i, r)$ holds for all sufficiently large $r \in \mathbb{Z}^{+}$.

As assertions $1^{\prime \prime}$ and $2^{\prime \prime}$ are together equivalent to our Claim, the proof is complete.

Our next lemma is equivalent to a result that was stated and used in BDHK07] and is a generalization of the following well-known fact, which says that the operators $P_{i}$ are nonexpansive:

Fact 5.4. For any index $i$ and all $y, z \in \mathbb{R}^{n},\left\|P_{i}(y)-P_{i}(z)\right\| \leq\|y-z\|$.

Fact 5.4 follows from Thm. 3.6 in [GoRe84, Ch. 1]; it can be deduced from the fact that if $P_{i}(y) \neq P_{i}(z)$ then (as a consequence of Lemma 2.2) $y$ and $z$ must lie on opposite sides of the open set bounded by the hyperplanes orthogonal to the vector $P_{i}(y)-P_{i}(z)$ that contain $P_{i}(y)$ and $P_{i}(z)$.

Lemma 5.5. Let $y, z \in \mathbb{R}^{n}$ and let $w$ be an arbitrary weight function. Then:

$$
\left\|\sum_{\iota \in \mathbb{I}} w(\iota) P[\iota](y)-\sum_{\iota \in \mathbb{I}} w(\iota) P[\iota](z)\right\| \leq\|y-z\|
$$

In particular, we have that $\left\|T_{k}(y)-T_{k}(z)\right\| \leq\|y-z\|$ for all iteration numbers $k$.

Proof. It follows easily from Fact 5.4 that:

$$
\|P[\iota](y)-P[\iota](z)\| \leq\|y-z\| \quad \text { for all } \iota \in \mathbb{I} .
$$

Hence we have that

$$
\begin{array}{rlr}
\| \sum_{\iota \in \mathbb{I}} w(\iota) P[\iota](y)- & \sum_{\iota \in \mathbb{I}} w(\iota) P[\iota](z) \| & \\
& =\left\|\sum_{\iota \in \mathbb{I}} w(\iota)(P[\iota](y)-P[\iota](z))\right\| & \\
& \leq \sum_{\iota \in \mathbb{I}} w(\iota)\|P[\iota](y)-P[\iota](z)\| & \text { by the triangle inequality } \\
& \leq \sum_{\iota \in \mathbb{I}} w(\iota)\|y-z\| & \text { by (5.7) } \\
& =\|y-z\| & \text { as } \sum_{\iota \in \mathbb{I}} w(\iota)=1
\end{array}
$$

as $\sum_{\iota \in \mathbb{I}} w(\iota)=1$

which proves the lemma.

When the hypotheses of Lemma 5.2 or Lemma 5.3 hold and $l$ is a nonnegative integer, we define $x_{l}^{\infty}$ by:

$$
x_{l}^{\infty}=\lim _{k \rightarrow \infty}\left(T_{k-1} \circ \cdots \circ T_{l}\right) x_{*}^{l}=\lim _{k \rightarrow \infty} x_{l}^{k}
$$

The existence of the limit $x_{l}^{\infty}$ is guaranteed by the two lemmas. Now we are ready to state and prove the following proposition, which contains our Fourth Convergence Theorem:

Proposition 5.6. Suppose at least one of the following is true:

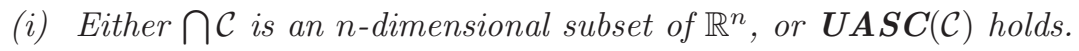


(ii) $w^{0}, w^{1}, w^{2}, \ldots$ satisfy conditions $\boldsymbol{C 1}$ and $\boldsymbol{C 2}$ of the Second Convergence Theorem for some sequence $f_{1}, f_{2}, f_{3}, \cdots \in[0,1]$ such that $\sum_{r=1}^{\infty} f_{r}=\infty$ and some sequence of iteration numbers $\kappa_{1}<\kappa_{2}<\kappa_{3}<\ldots$

Then there exists a point $x_{\infty}^{\infty}$ such that all of the following are true:

1. $\lim _{k \rightarrow \infty} x_{*}^{k}=\lim _{l \rightarrow \infty} x_{l}^{\infty}=x_{\infty}^{\infty}$

2. For $k \geq l \geq 0,\left\|x_{*}^{k}-x_{\infty}^{\infty}\right\| \leq\left\|\left(T_{k-1} \circ \cdots \circ T_{l}\right) x_{*}^{l}-x_{l}^{\infty}\right\|+2 \sum_{j=l}^{\infty}\left\|\boldsymbol{v}^{j}\right\|=\left\|x_{l}^{k}-x_{l}^{\infty}\right\|+2 \sum_{j=l}^{\infty}\left\|\boldsymbol{v}^{j}\right\|$.

3. If (i) holds, then $x_{\infty}^{\infty} \in C_{j}$ for every index $j$ that satisfies $\sum_{k=0}^{\infty} \sum_{\iota \in \mathbb{I}\langle j\rangle} w^{k}(\iota)=\infty$. If (ii) holds, then $x_{\infty}^{\infty} \in \bigcap \mathcal{C}$.

Proof. As $x_{l+1}^{l+1}-x_{l}^{l+1}=\boldsymbol{v}^{l}$ for all $l \geq 0$, we see from (5.4) (or Fig. 5.1) and Lemma 5.5 that

$$
\left\|x_{l}^{k}-x_{l+1}^{k}\right\| \leq\left\|\boldsymbol{v}^{l}\right\| \quad \text { whenever } k>l \geq 0
$$

From this and the triangle inequality we deduce that

$$
\left\|x_{l}^{k}-x_{m}^{k}\right\|=\left\|\sum_{j=l}^{m-1}\left(x_{j}^{k}-x_{j+1}^{k}\right)\right\| \leq \sum_{j=l}^{m-1}\left\|x_{j}^{k}-x_{j+1}^{k}\right\| \leq \sum_{j=l}^{m-1}\left\|\boldsymbol{v}^{j}\right\| \text { whenever } k \geq m \geq l \geq 0 .
$$

It follows from (5.9) that:

$$
\begin{array}{ll}
\left\|x_{l}^{\infty}-x_{m}^{\infty}\right\| \leq \sum_{j=l}^{m-1}\left\|\boldsymbol{v}^{j}\right\| \leq \sum_{j=l}^{\infty}\left\|\boldsymbol{v}^{j}\right\| & \text { whenever } m \geq l \geq 0 . \\
\left\|x_{*}^{k}-x_{l}^{k}\right\|=\left\|x_{k}^{k}-x_{l}^{k}\right\| \leq \sum_{j=l}^{k-1}\left\|\boldsymbol{v}^{j}\right\| \leq \sum_{j=l}^{\infty}\left\|\boldsymbol{v}^{j}\right\| & \text { whenever } k \geq l \geq 0 . \\
\left\|x_{*}^{k}-x_{m}^{\infty}\right\| \leq\left\|x_{l}^{k}-x_{l}^{\infty}\right\|+2 \sum_{j=l}^{\infty}\left\|\boldsymbol{v}^{j}\right\| & \text { whenever } \min (m, k) \geq l \geq 0 .
\end{array}
$$

Here (5.12) follows from (5.10), (5.11), and the triangle inequality. Since $\sum_{j=l}^{\infty}\left\|\boldsymbol{v}^{j}\right\| \rightarrow 0$ as $l \rightarrow \infty$, we see from (5.10) that $\left(x_{l}^{\infty}\right)_{l=0}^{\infty}$ is a Cauchy sequence. Let $x_{\infty}^{\infty}$ be the limit of this sequence, so the second equality of statement 1 holds. Then statement 2 also holds, because of (5.12) and the fact that $\lim _{m \rightarrow \infty} x_{m}^{\infty}=x_{\infty}^{\infty}$.

For every $\epsilon>0$, let $l(\epsilon)$ and $K(\epsilon)$ be integers such that $\sum_{j=l(\epsilon)}^{\infty}\left\|\boldsymbol{v}^{j}\right\|<\epsilon / 3$ and $\left\|x_{l(\epsilon)}^{k}-x_{l(\epsilon)}^{\infty}\right\|<\epsilon / 3$ whenever $k>K(\epsilon)$. Then for every $\epsilon>0$ we see (on putting $l=l(\epsilon)$ in statement 2) that $\left\|x_{*}^{k}-x_{\infty}^{\infty}\right\|<\epsilon$ whenever $k>K(\epsilon)$. This establishes statement 1 .

In case (i), Lemma 5.2 tells us $x_{l}^{\infty} \in C_{j}$ for every $l$ and every index $j$ such that $\sum_{k=0}^{\infty} \sum_{\iota \in \mathbb{I}\langle j\rangle} w^{k}(\iota)=\infty$. This and statement 1 imply $x_{\infty}^{\infty} \in C_{j}$ for every index $j$ such that $\sum_{k=0}^{\infty} \sum_{\iota \in \mathbb{I}\langle j\rangle} w^{k}(\iota)=\infty$ (as $C_{j}$ is closed for every $j$ ). In case (ii), Lemma 5.3 tells us $x_{l}^{\infty} \in \bigcap \mathcal{C}$ for every $l$. This and statement 1 imply $x_{\infty}^{\infty} \in \bigcap \mathcal{C}$ (as $\cap \mathcal{C}$ is closed). Hence statement 3 holds.

As mentioned earlier, our Fourth Convergence Theorem follows immediately from statements 1 and 3 of the above proposition.

Statement 2 of the proposition is equivalent to the case of [BaRZ18, eqn. (4.5)] in which the Hilbert space $\mathcal{H}$, sequence $\left(e_{k}\right)_{k=i}^{\infty}$, and points $x^{k+1}, x^{i}$, and $x^{\infty}$ of [BaRZ18, eqn. (4.5)] are respectively $\mathbb{R}^{n}$, our sequence $\left(\left\|\boldsymbol{v}^{k}\right\|\right)_{k=i}^{\infty}$, and our points $x_{*}^{k+1}, x_{*}^{i}$, and $x_{\infty}^{\infty}$. (However, in case (i) of the proposition our points $x_{l}^{\infty}$ need not satisfy the hypothesis of [BaRZ18, eqn. (4.5)] that these points be common fixed points of all the $T_{k} \mathrm{~s}$.) It follows from statement 2 that, under the hypotheses of either part of the Fourth Convergence Theorem, the perturbations of (5.1) preserve the rate of convergence of the iterative scheme (1.1) in a sense that is explained and illustrated in Remark 13 and Example 16 of BaRZ18. This applies, in particular, to perturbations introduced by superiorization of the iterative scheme (1.1) [BaRZ18, Cor. 14]. 


\section{Fifth Convergence Theorem}

Recall from sec. 1.2 that the operators $P_{i}^{\epsilon}$ and $P^{\epsilon}[\iota]$ are defined for every $\epsilon>0$, every index $i$, and every index vector $\iota=\left(\iota_{1}, \iota_{2}, \ldots, \iota_{m}\right)$ as follows:

$$
\begin{aligned}
P_{i}^{\epsilon}(x) & = \begin{cases}P_{i}(x) & \text { if }\left\|x-P_{i}(x)\right\| \geq \epsilon \\
x & \text { if }\left\|x-P_{i}(x)\right\|<\epsilon\end{cases} \\
P^{\epsilon}[\iota] & =P_{\iota_{m}}^{\epsilon} \circ P_{\iota_{m-1}}^{\epsilon} \circ \cdots \circ P_{\iota_{1}}^{\epsilon}
\end{aligned}
$$

Our final convergence theorem deals with sequences that are generated using the operators $P^{\epsilon}[\iota]$ rather than the operators $P[\iota]$.

Theorem 6.1 (Fifth Convergence Theorem). Let $x_{\epsilon}^{0}$ be any point in $\mathbb{R}^{n}$, and let $x_{\epsilon}^{1}, x_{\epsilon}^{2}, x_{\epsilon}^{3} \cdots \in \mathbb{R}^{n}$ be the sequence of points defined by

$$
x_{\epsilon}^{k+1}=\left(\sum_{\iota \in \mathbb{I}} w^{k}(\iota) P^{\epsilon}[\iota]\left(x_{\epsilon}^{k}\right)\right)+\boldsymbol{v}^{k} \quad \text { for all } k \geq 0,
$$

where $\boldsymbol{v}^{0}, \boldsymbol{v}^{1}, \boldsymbol{v}^{2}, \ldots$ is a sequence of vectors in $\mathbb{R}^{n}$ such that $\sum_{k=0}^{\infty}\left\|\boldsymbol{v}^{k}\right\|$ converges. Then the sequence $x_{\epsilon}^{1}, x_{\epsilon}^{2}, x_{\epsilon}^{3}, \ldots$ converges. Moreover, if $p$ is the limit of this sequence then $d\left(p, C_{j}\right) \leq \epsilon$ for every index $j$ such that $\sum_{k=0}^{\infty} \sum_{\iota \in \mathbb{I}\langle j\rangle} w^{k}(\iota)=\infty$.

We mention here that Fact 5.4 does not hold when $P_{i}$ is replaced by $P_{i}^{\epsilon}$ : When $C_{i} \neq \mathbb{R}^{n}$ the operator $P_{i}^{\epsilon}$ is not non-expansive; indeed, $P_{i}^{\epsilon}$ is not even continuous at those points $x$ for which $d\left(x, C_{i}\right)=\epsilon$. So the argument we used to prove the Fourth Convergence Theorem cannot be used to deduce the Fifth Convergence Theorem from the special case of the theorem in which $\boldsymbol{v}^{k}=0$ for every $k$.

\subsection{Proof of the Fifth Convergence Theorem}

For every weight function $w$, index vector $\iota$, and point $x \in \mathbb{R}^{n}$ we define the following complementary sets of index vectors:

$$
\begin{aligned}
& \mathbb{I}_{\epsilon}(x)=\left\{\iota \in \mathbb{I} \mid d\left(x, C_{j}\right)<\epsilon \text { for all } j \in \operatorname{IndexSet}(\iota)\right\} \\
& \overline{\mathbb{I}}_{\epsilon}(x)=\left\{\iota \in \mathbb{I} \mid d\left(x, C_{j}\right) \geq \epsilon \text { for at least one } j \in \operatorname{IndexSet}(\iota)\right\}=\mathbb{I} \backslash \mathbb{I}_{\epsilon}(x)
\end{aligned}
$$

Lemma 6.2. Let $c$ be a point in $\bigcap \mathcal{C}, x$ a point in $\mathbb{R}^{n}$, and $i$ an index. Then:

1. $\left\|P_{i}^{\epsilon}(x)-c\right\|^{2} \leq\|x-c\|^{2}-\epsilon^{2}$ if $d\left(x, C_{i}\right) \geq \epsilon$.

2. $\left\|P_{i}^{\epsilon}(x)-c\right\| \leq\|x-c\|$, with equality if and only if $d\left(x, C_{i}\right)<\epsilon$.

Corollary 6.3. Let $c$ be a point in $\bigcap \mathcal{C}, x$ a point in $\mathbb{R}^{n}$, and $\iota$ an index vector. Then we have that $\left\|P^{\epsilon}[\iota](x)-c\right\|^{2} \leq\|x-c\|^{2}-\epsilon^{2}$ if $\iota \in \overline{\mathbb{I}_{\epsilon}}(x)$.

Corollary 6.4. Let $c$ be a point in $\bigcap \mathcal{C}, x$ a point in $\mathbb{R}^{n}$, and $\iota$ an index vector. Then $\left\|P^{\epsilon}[\iota](x)-c\right\| \leq\|x-c\|$, with equality if and only if $\iota \in \mathbb{I}_{\epsilon}(x)$.

Proof of Lemma 6.2. Assertion 1 follows from Proposition 2.3. because if $d\left(x, C_{i}\right) \geq \epsilon$ then $P_{i}^{\epsilon}(x)=P_{i}(x)$.

If $d\left(x, C_{i}\right)<\epsilon$, then $\left\|P_{i}^{\epsilon}(x)-c\right\|=\|x-c\|$ (because $P_{i}^{\epsilon}(x)=x$ ) and so assertion 2 is true. If $d\left(x, C_{i}\right) \geq \epsilon$, then assertion 1 implies $\left\|P_{i}^{\epsilon}(x)-c\right\|<\|x-c\|$ and so assertion 2 is again true.

Using Corollary 6.4, we show:

Lemma 6.5. Let $c$ be a point in $\bigcap \mathcal{C}$. Then:

$$
\begin{aligned}
\left\|x_{\epsilon}^{k+1}-c\right\| & \leq\left\|x_{\epsilon}^{k}-c\right\|+\left\|\boldsymbol{v}^{k}\right\| \quad \text { for any iteration number } k . \\
\left\|x_{\epsilon}^{k}-c\right\| & \leq\left\|x_{\epsilon}^{0}-c\right\|+\sum_{\ell=0}^{k-1}\left\|\boldsymbol{v}^{\ell}\right\| \quad \text { for any iteration number } k .
\end{aligned}
$$


Proof. The first inequality holds because

$$
\begin{aligned}
\left\|x_{\epsilon}^{k+1}-c\right\| & =\left\|\left(\sum_{\iota \in \mathbb{I}} w^{k}(\iota) P^{\epsilon}[\iota]\left(x_{\epsilon}^{k}\right)\right)+\boldsymbol{v}^{k}-c\right\| & & \\
& \leq\left\|\boldsymbol{v}^{k}\right\|+\left\|\left(\sum_{\iota \in \mathbb{I}} w^{k}(\iota) P^{\epsilon}[\iota]\left(x_{\epsilon}^{k}\right)\right)-c\right\| & & \text { by the triangle inequality } \\
& \leq\left\|\boldsymbol{v}^{k}\right\|+\sum_{\iota \in \mathbb{I}} w^{k}(\iota)\left\|P^{\epsilon}[\iota]\left(x_{\epsilon}^{k}\right)-c\right\| & & \text { as } z \mapsto\|z-c\| \text { is convex } \\
& \leq\left\|\boldsymbol{v}^{k}\right\|+\sum_{\iota \in \mathbb{I}} w^{k}(\iota)\left\|x_{\epsilon}^{k}-c\right\| & & \text { by Corollary 6.4 } \\
& =\left\|\boldsymbol{v}^{k}\right\|+\left\|x_{\epsilon}^{k}-c\right\| & & \text { as } \sum_{\iota \in \mathbb{I}} w^{k}(\iota)=1
\end{aligned}
$$

The second inequality follows from the first.

In the sequel $M_{\star}$ will denote the nonnegative number defined by

$$
M_{\star}=2\left(d\left(x_{\epsilon}^{0}, \bigcap \mathcal{C}\right)+\sum_{k=0}^{\infty}\left\|\boldsymbol{v}^{k}\right\|\right)
$$

We observe that $M_{\star}$ depends only on the seed point $x_{\epsilon}^{0}$, the collection $\mathcal{C}$, and the sum $\sum_{k=0}^{\infty}\left\|\boldsymbol{v}^{k}\right\|$ of the lengths of the perturbation vectors.

Lemma 6.6. Let $\iota$ be any index vector and $k$ any iteration number. Then:

$$
\left\|P^{\epsilon}[\iota]\left(x_{\epsilon}^{k}\right)+\boldsymbol{v}^{k}-x_{\epsilon}^{k}\right\| \leq M_{\star}
$$

Proof. For every $c \in \cap \mathcal{C}$ we have that:

$$
\begin{aligned}
\left\|P^{\epsilon}[\iota]\left(x_{\epsilon}^{k}\right)+\boldsymbol{v}^{k}-x_{\epsilon}^{k}\right\| & \leq\left\|\boldsymbol{v}^{k}\right\|+\left\|P^{\epsilon}[\iota]\left(x_{\epsilon}^{k}\right)-c\right\|+\left\|c-x_{\epsilon}^{k}\right\| & & \text { by the triangle inequality } \\
& \leq\left\|\boldsymbol{v}^{k}\right\|+\left\|x_{\epsilon}^{k}-c\right\|+\left\|c-x_{\epsilon}^{k}\right\| & & \text { by Corollary 6.4 } \\
& =\left\|\boldsymbol{v}^{k}\right\|+2\left\|x_{\epsilon}^{k}-c\right\| & & \\
& \leq\left\|\boldsymbol{v}^{k}\right\|+2\left(\left\|x_{\epsilon}^{0}-c\right\|+\sum_{\ell=0}^{k-1}\left\|\boldsymbol{v}^{\ell}\right\|\right) & & \text { by Lemma 6.5 } \\
& \leq 2\left(\left\|x_{\epsilon}^{0}-c\right\|+\sum_{\ell=0}^{\infty}\left\|\boldsymbol{v}^{\ell}\right\|\right) & &
\end{aligned}
$$

Hence $\left\|P^{\epsilon}[\iota]\left(x_{\epsilon}^{k}\right)+\boldsymbol{v}^{k}-x_{\epsilon}^{k}\right\| \leq \inf _{c \in \mathcal{C}} 2\left(\left\|x_{\epsilon}^{0}-c\right\|+\sum_{\ell=0}^{\infty}\left\|\boldsymbol{v}^{\ell}\right\|\right)=M_{\star}$.

The sums $\sum_{\iota \in \mathbb{I}_{\epsilon}\left(x_{\epsilon}^{k}\right)} w^{k}(\iota)$ and $\sum_{\iota \in \overline{\mathbb{I}}_{\epsilon}\left(x_{\epsilon}^{k}\right)} w^{k}(\iota)$ will be much used in the sequel. These are respectively the sum of the weights at iteration $k$ of those operators $P^{\epsilon}[\iota]$ for which $P^{\epsilon}[\iota]\left(x_{\epsilon}^{k}\right)=x_{\epsilon}^{k}$, and the sum of the weights at iteration $k$ of those operators $P^{\epsilon}[\iota]$ for which $P^{\epsilon}[\iota]\left(x_{\epsilon}^{k}\right) \neq x_{\epsilon}^{k}$. (As the functions $w^{0}, w^{1}, w^{2}, \ldots$ need not be fixed in advance, $w^{k}$ may be chosen after computing $P^{\epsilon}[\iota]\left(x_{\epsilon}^{k}\right)$ for various index vectors $\iota$, in which case it would be quite natural to choose $w^{k}$ so that either $w^{k}(\iota)=0$ for all $\iota \in \mathbb{I}_{\epsilon}\left(x_{\epsilon}^{k}\right)$ and $\sum_{\iota \in \overline{\mathbb{I}}_{\epsilon}\left(x_{\epsilon}^{k}\right)} w^{k}(\iota)=1$, or $x_{\epsilon}^{k+1}=x_{\epsilon}^{k}$. However, we will not assume this.)

For any iteration numbers $r_{1}<r_{2}$ and any $s$ in $\mathbb{R}^{n}$, the next lemma gives an upper bound for $\left\|x_{\epsilon}^{r_{2}}-x_{\epsilon}^{r_{1}}\right\|$ in terms of $\sum_{k=r_{1}}^{r_{2}-1} \sum_{\iota \in \overline{\mathbb{I}}_{\epsilon}\left(x_{\epsilon}^{k}\right)} w^{k}(\iota)$.

Lemma 6.7. Let $r_{1}$ and $r_{2}$ be iteration numbers such that $r_{1}<r_{2}$, and let $s \in \mathbb{R}^{n}$. Then we have that $\left\|x_{\epsilon}^{r_{2}}-x_{\epsilon}^{r_{1}}\right\| \leq \sum_{k=r_{1}}^{r_{2}-1}\left\|\boldsymbol{v}^{k}\right\|+M_{\star} \sum_{k=r_{1}}^{r_{2}-1} \sum_{\iota \in \overline{\mathbb{I}}_{\epsilon}\left(x_{\epsilon}^{k}\right)} w^{k}(\iota)$. 
Proof. For any iteration number $k$, it follows from the convexity of $z \mapsto\|z+u\|$ (for any $u \in \mathbb{R}^{n}$ ), the fact that $P^{\epsilon}[\iota]\left(x_{\epsilon}^{k}\right)=x_{\epsilon}^{k}$ when $\iota \in \mathbb{I}_{\epsilon}\left(x_{\epsilon}^{k}\right)$, and Lemma 6.6 that:

$$
\begin{array}{rlrl}
\left\|x_{\epsilon}^{k+1}-x_{\epsilon}^{k}\right\| & =\left\|\left(\sum_{\iota \in \mathbb{I}} w^{k}(\iota) P^{\epsilon}[\iota]\left(x_{\epsilon}^{k}\right)\right)+\boldsymbol{v}^{k}-x_{\epsilon}^{k}\right\| & & \\
& \leq \sum_{\iota \in \mathbb{I}} w^{k}(\iota)\left\|P^{\epsilon}[\iota]\left(x_{\epsilon}^{k}\right)+\boldsymbol{v}^{k}-x_{\epsilon}^{k}\right\| & & \text { as } z \mapsto\left\|z+\boldsymbol{v}^{k}-x_{\epsilon}^{k}\right\| \text { is convex } \\
& =\sum_{\iota \in \mathbb{I}_{\epsilon}\left(x_{\epsilon}^{k}\right)} w^{k}(\iota)\left\|P^{\epsilon}[\iota]\left(x_{\epsilon}^{k}\right)+\boldsymbol{v}^{k}-x_{\epsilon}^{k}\right\| & & \\
& \quad+\sum_{\iota \in \mathbb{\mathbb { I }}_{\epsilon}\left(x_{\epsilon}^{k}\right)} w^{k}(\iota)\left\|P^{\epsilon}[\iota]\left(x_{\epsilon}^{k}\right)+\boldsymbol{v}^{k}-x_{\epsilon}^{k}\right\| & \\
\leq & \sum_{\iota \in \mathbb{I}_{\epsilon}\left(x_{\epsilon}^{k}\right)} w^{k}(\iota)\left\|\boldsymbol{v}^{k}\right\|+\sum_{\iota \in \overline{\mathbb{I}}_{\epsilon}\left(x_{\epsilon}^{k}\right)} w^{k}(\iota) M_{\star} & & \text { by Lemma }[6.6 \\
& \leq\left\|\boldsymbol{v}^{k}\right\|+\sum_{\iota \in \overline{\mathbb{I}}_{\epsilon}\left(x_{\epsilon}^{k}\right)} w^{k}(\iota) M_{\star} & & \text { as } \sum_{\iota \in \mathbb{I}_{\epsilon}\left(x_{\epsilon}^{k}\right)} w^{k}(\iota) \leq 1
\end{array}
$$

The lemma follows from this and the triangle inequality.

Lemma 6.8. Let $c$ be any point in $\bigcap \mathcal{C}$. Then there exists a number $A$ that is independent of $k$ such that $\left\|x_{\epsilon}^{k+1}-c\right\|^{2} \leq\left\|x_{\epsilon}^{k}-c\right\|^{2}-\epsilon^{2} \sum_{\iota \in \overline{\mathbb{I}}_{\epsilon}\left(x_{\epsilon}^{k}\right)} w^{k}(\iota)+A\left\|\boldsymbol{v}^{k}\right\|$ for every iteration number $k$.

Corollary 6.9. Let $c$ be any point in $\bigcap \mathcal{C}$, Then there exists a number $A$ that is independent of $\kappa$ such that $\left\|x_{\epsilon}^{\kappa}-c\right\|^{2} \leq\left\|x_{\epsilon}^{0}-c\right\|^{2}-\epsilon^{2} \sum_{k=0}^{\kappa-1} \sum_{\iota \in \overline{\mathbb{I}}_{\epsilon}\left(x_{\epsilon}^{k}\right)} w^{k}(\iota)+A \sum_{k=0}^{\infty}\left\|\boldsymbol{v}^{k}\right\|$ for every iteration number $\kappa$.

Corollary 6.10. The series $\sum_{k=0}^{\infty} \sum_{\iota \in \overline{\mathbb{I}}_{\epsilon}\left(x_{\epsilon}^{k}\right)} w^{k}(\iota)$ converges.

Proof of Lemma 6.8. Let $A=2\left(\left\|x_{\epsilon}^{0}-c\right\|+\sum_{\ell=0}^{\infty}\left\|\boldsymbol{v}^{\ell}\right\|\right)$, and let $k$ be any iteration number. Then:

$$
\begin{aligned}
\left\|x_{\epsilon}^{k+1}-c\right\|^{2}= & \left\|\left(\sum_{\iota \in \mathbb{I}} w^{k}(\iota) P^{\epsilon}[\iota]\left(x_{\epsilon}^{k}\right)\right)+\boldsymbol{v}^{k}-c\right\|^{2} & \\
\leq & \sum_{\iota \in \mathbb{I}} w^{k}(\iota)\left\|P^{\epsilon}[\iota]\left(x_{\epsilon}^{k}\right)+\boldsymbol{v}^{k}-c\right\|^{2} & \text { as } z \mapsto\left\|z+\boldsymbol{v}^{k}-c\right\|^{2} \text { is convex } \\
\leq & \sum_{\iota \in \mathbb{I}} w^{k}(\iota)\left(\left\|\boldsymbol{v}^{k}\right\|+\left\|P^{\epsilon}[\iota]\left(x_{\epsilon}^{k}\right)-c\right\|\right)^{2} & \text { by the triangle inequality } \\
= & \sum_{\iota \in \mathbb{I}} w^{k}(\iota)\left(\left\|\boldsymbol{v}^{k}\right\|^{2}+2\left\|\boldsymbol{v}^{k}\right\|\left\|P^{\epsilon}[\iota]\left(x_{\epsilon}^{k}\right)-c\right\|\right) & \\
& +\sum_{\iota \in \mathbb{I}} w^{k}(\iota)\left\|P^{\epsilon}[\iota]\left(x_{\epsilon}^{k}\right)-c\right\|^{2} &
\end{aligned}
$$

The first of the two sums on the right side of (6.4) satisfies

$$
\begin{array}{rlrl}
\sum_{\iota \in \mathbb{I}} w^{k}(\iota)\left(\left\|\boldsymbol{v}^{k}\right\|^{2}\right. & \left.+2\left\|\boldsymbol{v}^{k}\right\|\left\|P^{\epsilon}[\iota]\left(x_{\epsilon}^{k}\right)-c\right\|\right) & \\
& \leq \sum_{\iota \in \mathbb{I}} w^{k}(\iota)\left(\left\|\boldsymbol{v}^{k}\right\|^{2}+2\left\|\boldsymbol{v}^{k}\right\|\left\|x_{\epsilon}^{k}-c\right\|\right) & & \text { by Corollary 6.4 } \\
& =\left\|\boldsymbol{v}^{k}\right\|^{2}+2\left\|\boldsymbol{v}^{k}\right\|\left\|x_{\epsilon}^{k}-c\right\| & & \text { as } \sum_{\iota \in \mathbb{I}} w^{k}(\iota)=1 \\
& =\left(\left\|\boldsymbol{v}^{k}\right\|+2\left\|x_{\epsilon}^{k}-c\right\|\right)\left\|\boldsymbol{v}^{k}\right\| & \\
& \leq\left(\left\|\boldsymbol{v}^{k}\right\|+2\left(\left\|x_{\epsilon}^{0}-c\right\|+\sum_{\ell=0}^{k-1}\left\|\boldsymbol{v}^{\ell}\right\|\right)\right)\left\|\boldsymbol{v}^{k}\right\| & & \text { by } \\
& \leq A\left\|\boldsymbol{v}^{k}\right\|
\end{array}
$$

The second of the two sums on the right side of (6.4) satisfies

$$
\begin{aligned}
\sum_{\iota \in \mathbb{I}} w^{k}(\iota)\left\|P^{\epsilon}[\iota]\left(x_{\epsilon}^{k}\right)-c\right\|^{2}= & \sum_{\iota \in \mathbb{I}_{\epsilon}\left(x_{\epsilon}^{k}\right)} w^{k}(\iota)\left\|P^{\epsilon}[\iota]\left(x_{\epsilon}^{k}\right)-c\right\|^{2} \\
& +\sum_{\iota \in \mathbb{I}_{\epsilon}}\left(x_{\epsilon}^{k}\right) w^{k}(\iota)\left\|P^{\epsilon}[\iota]\left(x_{\epsilon}^{k}\right)-c\right\|^{2} \\
\leq & \sum_{\iota \in \mathbb{I}_{\epsilon}\left(x_{\epsilon}^{k}\right)} w^{k}(\iota)\left\|x_{\epsilon}^{k}-c\right\|^{2} \\
& +\sum_{\iota \in \mathbb{\mathbb { I }}_{\epsilon}\left(x_{\epsilon}^{k}\right)} w^{k}(\iota)\left(\left\|x_{\epsilon}^{k}-c\right\|^{2}-\epsilon^{2}\right) \quad \text { by Corollary } 6.3 \\
= & \left\|x_{\epsilon}^{k}-c\right\|^{2}-\epsilon^{2} \sum_{\iota \in \overline{\mathbb{I}}_{\epsilon}\left(x_{\epsilon}^{k}\right)} w^{k}(\iota) \quad \text { as } \sum_{\iota \in \mathbb{I}} w^{k}(\iota)=1
\end{aligned}
$$


The lemma follows from this, (6.4), and (6.5).

Completion of the Proof of the Fifth Convergence Theorem. As $\sum_{k=0}^{\infty} \sum_{\iota \in \overline{\mathbb{I}}_{\epsilon}}\left(x_{\epsilon}^{k}\right) w^{k}(\iota)$ converges (by Corollary 6.10), it follows from Lemma 6.7 that $x_{\epsilon}^{0}, x_{\epsilon}^{1}, x_{\epsilon}^{2}, \ldots$ is a Cauchy sequence and must therefore converge.

Let $p$ be the limit of the sequence, and let $j$ be any index such that $d\left(p, C_{j}\right)>\epsilon$. To complete the proof of the theorem, we show that the series $\sum_{k=0}^{\infty} \sum_{\iota \in \mathbb{I}\langle j\rangle} w^{k}(\iota)$ converges.

Since $p$ is the limit of $x_{\epsilon}^{0}, x_{\epsilon}^{1}, x_{\epsilon}^{2}, \ldots$, we see from the definition of $j$ that $d\left(x_{\epsilon}^{k}, C_{j}\right)>\epsilon$ for all sufficiently large iteration numbers $k$. Hence $\mathbb{I}\langle j\rangle \subseteq \overline{\mathbb{I}}_{\epsilon}\left(x_{\epsilon}^{k}\right)$ for all sufficiently large $k$. So the fact that $\sum_{k=0}^{\infty} \sum_{\iota \in \overline{\mathbb{I}}_{\epsilon}\left(x_{\epsilon}^{k}\right)} w^{k}(\iota)$ converges implies $\sum_{k=0}^{\infty} \sum_{\iota \in \mathbb{I}\langle j\rangle} w^{k}(\iota)$ also converges.

\section{Discussion And Conclusion}

For any infinite sequence of closed convex sets $\left(C_{i}\right)_{i=1}^{\infty}$ in $\mathbb{R}^{n}$ such that $\bigcap_{i=1}^{\infty} C_{i} \neq \emptyset$, a string-averaging algorithm based on the corresponding projection operators $\left(P_{i}: \mathbb{R}^{n} \rightarrow C_{i}\right)_{i=1}^{\infty}$ generates a sequence of iterates $\left(x^{k}\right)_{k=0}^{\infty}$ in which each iterate $x^{k+1}$ is a weighted average of the results of applying certain finite sequences of those projections (i.e., certain finite sequences of $P_{i} \mathrm{~s}$ ) to $x^{k}$. This paper addresses the problem of finding sufficient conditions (on $\left(C_{i}\right)_{i=1}^{\infty}$ and/or on the averaging weights and the sequences of $P_{i}$ s that are used) for $\left(x^{k}\right)_{k=0}^{\infty}$ to converge to a point in $\bigcap_{i=1}^{\infty} C_{i}$.

In most prior work, string-averaging algorithms have been defined for a finite sequence $\left(C_{i}\right)_{i=1}^{K}$ of closed convex sets rather than an infinite sequence. (Such algorithms can be understood as algorithms for the infinite sequence $\left(C_{i}\right)_{i=1}^{\infty}$ if we define $C_{i}=\mathbb{R}^{n}$ for all $i>K$.) In this finite case it is known-and follows from [ReZa16, Thm. 4.1] - that the iterates $\left(x^{k}\right)_{k=0}^{\infty}$ converge to a point in $\bigcap_{i=1}^{K} C_{i}$ if the three hypotheses stated as $\mathrm{H} 1-\mathrm{H} 3$ in sec. 1.2 are all satisfied.

The main focus of this paper is on the (countably) infinite case -i.e., the case where there are infinitely many indices $i$ for which $C_{i} \neq \mathbb{R}^{n}$. In this case it is impossible to satisfy H3, and the hypotheses H1 and $\mathrm{H} 2$ would be quite restrictive. Without assuming any of these additional hypotheses, we have proved three theorems that give sufficient conditions for the iterates $\left(x^{k}\right)_{k=0}^{\infty}$ to converge and for the iterates to converge to a point in $\bigcap_{i=1}^{\infty} C_{i}$.

From these results we have deduced a fourth theorem that generalizes the first three theorems to sequences of perturbed iterates for which the sum of the magnitudes of the perturbations converges. Such perturbed iterates are produced when an algorithm that generates $\left(x^{k}\right)_{k=1}^{\infty}$ is superiorized Cens15, CHJ17] to improve its efficacy. The fourth theorem implies that those algorithms whose iterates are shown by our first three theorems to converge to a point in $\bigcap_{i=1}^{\infty} C_{i}$ are also resilient to bounded perturbations, so that they are candidates for superiorization. When there are only finitely many indices $i$ for which $C_{i} \neq \mathbb{R}^{n}$ and the above-mentioned hypotheses $\mathrm{H} 1$ - H3 are satisfied, our fourth theorem is a known result that follows from ReZa16, Thm. 4.5].

Our fifth theorem deals with (possibly perturbed) sequences of iterates computed by algorithms that use operators $\left(P_{i}^{\epsilon}\right)_{i=1}^{\infty}$ rather than the operators $\left(P_{i}\right)_{i=1}^{\infty}$, where $P_{i}^{\epsilon}$ is defined as follows for any $\epsilon>0$ :

$$
P_{i}^{\epsilon}(x)= \begin{cases}P_{i}(x) & \text { if }\left\|x-P_{i}(x)\right\| \geq \epsilon \\ x & \text { if }\left\|x-P_{i}(x)\right\|<\epsilon\end{cases}
$$

Assuming that the sum of the magnitudes of any perturbations converges, this theorem implies that if the averaging weights satisfy a mild condition - namely that, for every index $i$ such that $C_{i} \neq \mathbb{R}^{n}$, the sum (over all iterations) of the weights of the index vectors that contain index $i$ is infinite - then the iterates converge to a point that is within distance $\epsilon$ of each of the $C_{i}$ s. We can gradually reduce $\epsilon$ - e.g., as explained at the end of sec. 1.2 - to obtain a sequence of iterates that can be shown (using Lemma 6.5) to converge to a point in $\bigcap_{i=1}^{\infty} C_{i}$ if the sum of the magnitudes of any perturbations used in computing this sequence converges. 


\section{ACKNOWLEDGMENTS}

The authors are grateful to a referee for detailed and helpful suggestions regarding the presentation of the

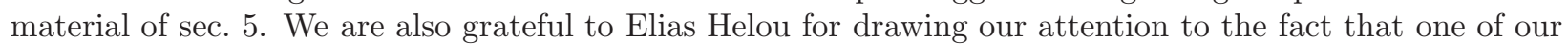
mathematical claims needed an additional hypothesis, and to Yair Censor, Touraj Nikazad, and two of the referees for bringing a number of references to our attention and for corrections to certain of our references.

\section{REFERENCES}

[AlCe05] A. Aleyner and Y. Censor, Best approximation to common fixed points of a semigroup of nonexpansive operators, J. Nonlinear Convex Anal., Vol. 6, 137-151, 2005.

[BaRZ18] C. Bargetz, S. Reich, and R. Zalas, Convergence properties of dynamic string-averaging projection methods in the presence of perturbations, Numer. Algor., Vol. 77, 185-209, 2018.

[BlHe06] D. Blat and A. O. Hero, III, Energy based sensor network source localization via projection onto convex sets (POCS), IEEE T. Signal Process., Vol. 54, 3614-3619, 2006.

[BDHK07] D. Butnariu, R. Davidi, G. T. Herman, and I. G. Kazantsev, Stable convergence behavior under summable perturbations of a class of projection methods for convex feasibility and optimization problems, IEEE J-STSP, Vol. 1, $540-547,2007$.

[BuRZ08] D. Butnariu, S. Reich, and A. J. Zaslavski, Stable convergence theorems for infinite products and powers of nonexpansive mappings, Numer. Funct. Anal. Optim., Vol. 29, 304-323, 2008.

[Cens15] Y. Censor, Weak and strong superiorization: between feasibility-seeking and minimization, An. Şt. Univ. Ovidius Constanţa, Vol. 23(3), 41-64, 2015.

[CDH10] Y. Censor, R. Davidi, and G. T. Herman, Perturbation resilience and superiorization of iterative algorithms, Inverse Probl., Vol. 26, 065008 (12pp), 2010.

[CeEl02] Y. Censor and T. Elfving, Block-iterative algorithms with diagonally scaled oblique projections for the linear feasibility problem, SIAM J. Matrix Anal. Appl., Vol. 24, 40-58, 2002.

[CEH01] Y. Censor, T. Elfving, and G.T. Herman, Averaging strings of sequential iterations for convex feasibility problems. Inherently Parallel Algorithms in Feasibility and Optimization and their Applications, D. Butnariu, Y. Censor, and S. Reich (Editors), Elsevier Science Publishers, Amsterdam, 101-114, 2001.

[CHJ17] Y. Censor, G. T. Herman, and M. Jiang (Editors), Special issue on superiorization: theory and applications, Inverse Probl., Vol. 33, No. 4, 2017.

[CeZa13] Y. Censor and A. J. Zaslavski, Convergence and perturbation resilience of dynamic string-averaging projection methods, Comput. Optim. Appl., Vol. 54, 65-76, 2013.

[CeZa15] Y. Censor and A. J. Zaslavski, Strict Fejér monotonicity by superiorization of feasibility-seeking projection methods, J. Optim. Theory Appl., Vol. 165, 172-187, 2015.

[Cimm38] G. Cimmino, Calcolo approssimato per le soluzioni dei sistemi di equazioni lineari, Ric. Sci. Progr. Tecn. Econom. Naz., Vol. 1, 326-333, 1938.

[Comb97] P. L. Combettes, Convex set theoretic image recovery by extrapolated iterations of parallel subgradient projections, IEEE T. Image Process., Vol. 6, 493-506, 1997.

[Comb97b] P. L. Combettes, Hilbertian convex feasibility problem: convergence of projection methods, Appl. Math. Optim., Vol. 35, 311-330, 1997.

[GoRe84] K. Goebel and S. Reich, Uniform Convexity, Hyperbolic Geometry, and Non-Expansive Mappings, Marcel Dekker, New York, 1984.

[GPR67] L. G. Gurin (Gubin), B. T. Polyak, and E.V. Raik, The method of projections for finding the common point of convex sets, USSR Comput. Math. Math. Phys., Vol. 7, Issue 6, 1-24, 1967.

[Kacz37] S. Kaczmarz, Angenäherte Auflösung von Systemen linearer Gleichungen, Bull. Int. Acad. Polon. Sci. A, 355-357, 1937.

[KSP09] I. G. Kazantsev, S. Schmidt, and H. F. Poulsen, A discrete spherical X-ray transform of orientation distribution functions using bounding cubes, Inverse Probl., Vol 25, 105009 (15pp), 2009.

[Nedi10] A. Nedić, Random projection algorithms for convex set intersection problems, 49th IEEE Conference on Decision and Control, 7655-7660, 2010.

[NiAb15] T. Nikazad and M. Abbasi, Perturbation-resilient iterative methods with an infinite pool of mappings, SIAM J. Numer. Anal., Vol. 53, 390-404, 2015.

[NiAb17] T. Nikazad and M. Abbasi, A unified treatment of some perturbed fixed point iterative methods with an infinite pool of operators, Inverse Probl., Vol. 33, 044002 (27pp), 2017. 
[PhSu13] W. Phuengrattana and S. Suantai, Common fixed points of an infinite family of nonexpansive mappings in uniformly convex metric spaces, Math. Comput. Model., Vol. 57, 306-310, 2013.

[ReZa16] S. Reich and R. Zalas, A modular string averaging procedure for solving the common fixed point problem for quasi-nonexpansive mappings in Hilbert space, Numer. Algor., Vol. 72, 297-323, 2016.

[Webs94] R. J. Webster, Convexity, Oxford University Press, Oxford, 1994.

[YaOg04] I. Yamada and N. Ogura, Adaptive projected subgradient method for asymptotic minimization of sequence of nonnegative convex functions, Numer. Funct. Anal. Optim., Vol. 25, 593-617, 2004.

[YYZ07] Y. Yao, J. Yao, and H. Zhou, Approximation methods for common fixed points of infinite countable family of nonexpansive mappings, Comput. Math. Appl., Vol. 53, 1380-1389, 2007.

Computer Science Department, Queens College, City University of New York, Flushing, Ny 11367, USA

E-mail address: tkong@qc.cuny.edu

Mathematics Department, Medgar Evers College, City University of New York, Brooklyn, Ny 11225, USA

E-mail address: hpajoohesh@mec.cuny.edu

Computer Science Ph.D. Program, The Graduate Center, City University of New York, New York, NY 10016, USA

E-mail address: gabortherman@yahoo.com 